Illinois State University

ISU ReD: Research and eData

Theses and Dissertations

4-26-2016

\title{
Alternative Pathway For Provision Of Acyl Coa Precursors For Fatty Acid Biosynthesis: Purification And Kinetic Characterization Of Phosphotransbutyrylase And Butyrate Kinase From Listeria Monocytogenes
}

Sirisha Sirobhushanam

Illinois State University, sirees78@gmail.com

Follow this and additional works at: https://ir.library.illinoisstate.edu/etd

Part of the Biochemistry Commons, Microbiology Commons, and the Molecular Biology Commons

\section{Recommended Citation}

Sirobhushanam, Sirisha, "Alternative Pathway For Provision Of Acyl Coa Precursors For Fatty Acid Biosynthesis: Purification And Kinetic Characterization Of Phosphotransbutyrylase And Butyrate Kinase From Listeria Monocytogenes" (2016). Theses and Dissertations. 637.

https://ir.library.illinoisstate.edu/etd/637

This Dissertation is brought to you for free and open access by ISU ReD: Research and eData. It has been accepted for inclusion in Theses and Dissertations by an authorized administrator of ISU ReD: Research and eData. For more information, please contact ISUReD@ilstu.edu. 


\title{
ALTERNATIVE PATHWAY FOR PROVISION OF ACYL COA PRECURSORS FOR FATTY ACID BIOSYNTHESIS: PURIFICATION AND KINETIC CHARACTERIZATION OF PHOSPHOTRANSBUTYRYLASE AND BUTYRATE KINASE FROM LISTERIA MONOCYTOGENES
}

\author{
Sirisha Sirobhushanam
}

\section{Pages}

Listeria monocytogenes is a foodborne pathogen that causes listeriosis, a disease characterized by gastroenteritis, meningitis, spontaneous miscarriages and high mortality rate among infected individuals. L. monocytogenes is a major concern in the food industry, due to its ability to grow at refrigeration temperatures and the zero tolerance policy of the FDA, resulting in expensive food recalls. Growth at low temperatures is aided in part by the high membrane content of branched-chain fatty acids (BCFAs) which imparts greater fluidity to the membrane. Mutants of L. monocytogenes impaired in BCFA biosynthesis display diminished growth at normal and low temperatures, exhibit lower tolerance to acidity and alkalinity, and demonstrate lower virulence. Addition of 2methylbutyrate, the source of the membrane BCFA anteiso C15:0, restores the membrane BCFA content and virulence factor expression. Supplementation with unnatural branched-chain carboxylic acids (BCCAs) such as 2- ethylbutyrate and 2- 
methylpentanoate results in the incorporation of novel BCFAs in the listerial membrane. Incorporation of supplemented carboxylic acids is evidence of their entry into the fatty acid biosynthesis pathway and thus a Bkd-independent pathway which catalyzes the conversion of these compounds into their activated CoA derivatives must exist in $L$. monocytogenes. We hypothesize that Ptb and Buk, the products of the first two genes of the $b k d$ operon, are involved in the sequential conversion of the supplemented BCCAs into their acyl CoA derivatives, which then presumably enter the fatty acid biosynthesis pathway for elongation. Ptb catalyzes the reversible conversion of acyl CoAs into acyl phosphates and Buk catalyzes the reversible phosphorylation of carboxylic acids. Ptb and Buk were heterologously expressed in E. coli, purified by affinity chromatography and utilized for analysis of their kinetic properties to determine their role in the activation of such carboxylic acids. Ptb and Buk both demonstrate broad substrate specificity and do not use acetate or hexanoate efficiently as substrates. Ptb and Buk exhibit a strong preference for substrates which have a chain length of C3-C5 thus indicating that they are not involved either in acetate metabolism or in the activation of long chain fatty acids. Ptb shows a strong preference for branched-chain substrates while Buk appears to demonstrate preference for BCCAs only with respect to $\mathrm{C} 3$ and $\mathrm{C} 4$ substrates. Both Ptb and Buk from L. monocytogenes demonstrate significant activity with unnatural BCCAs such as 2-ethylbutyrate and 2-methylpentanoate. Additionally, Buk exhibits substantial phosphorylation activity at low temperatures and appears to prefer BCCAs thus demonstrating a switch in substrate specificities at low temperatures. Ptb catalysis involves the formation of a ternary complex with acyl CoA and phosphate before release of the products. Similarly, Buk also forms a ternary complex with carboxylic acid and 
ATP before catalysis and release of products. Our work here indicates that Ptb and Buk likely act in a sequential manner in the activation and subsequent assimilation of exogenous straight chain carboxylic acids (SCCAs) and BCCAs.

KEYWORDS: Phosphotransbutyrylase, Butyrate kinase, Branched-chain carboxylic acids, Branched-chain fatty acids, Fatty acid biosynthesis, Membrane fatty acid composition 


\begin{abstract}
ALTERNATIVE PATHWAY FOR PROVISION OF ACYL COA PRECURSORS FOR FATTY ACID BIOSYNTHESIS: PURIFICATION AND KINETIC CHARACTERIZATION OF PHOSPHOTRANSBUTYRYLASE AND BUTYRATE KINASE FROM LISTERIA MONOCYTOGENES
\end{abstract}

SIRISHA SIROBHUSHANAM

A Dissertation Submitted in Partial Fulfillment of the Requirements for the Degree of

DOCTOR OF PHILOSOPHY

School of Biological Sciences

ILLINOIS STATE UNIVERSITY

2016 
(C) 2016 Sirisha Sirobhushanam 


\begin{abstract}
ALTERNATIVE PATHWAY FOR PROVISION OF ACYL COA PRECURSORS FOR FATTY ACID BIOSYNTHESIS: PURIFICATION AND KINETIC CHARACTERIZATION OF PHOSPHOTRANSBUTYRYLASE AND BUTYRATE KINASE FROM LISTERIA MONOCYTOGENES
\end{abstract}

\title{
SIRISHA SIROBHUSHANAM
}

COMMITTEE MEMBERS:

Brian J Wilkinson, Chair

Radheshyam Jayaswal

Craig Gatto

Laura A Vogel

Siqing Liu 


\section{ACKNOWLEDGMENTS}

I would like to express my deepest gratitude and appreciation for Dr. Brian J. Wilkinson, my Dissertation Chair, for giving me the courage to dream big and the confidence to follow through. His thought provoking ideas, generosity in sharing his vast knowledge, guidance and unfailing support, have made this dissertation possible. A special note of thanks to Dr. Craig Gatto for his time, expert opinion and guidance throughout this process.

I would also like to express my thanks and gratitude to my committee members Dr. R.K. Jayaswal, Dr. Laura Vogel and Dr. Siqing Liu. Their constructive ideas, counsel and support made my time at Illinois State University, one of learning and joy of discovery. I thank the Department of Biological Sciences, the Graduate School and the Phi Sigma Society for the excellent facilities, financial support and for fostering camaraderie and exchange of ideas among the different laboratories in the department. I would also like to thank Dr. Charitha Galva, and my lab mates, past and present, for their support and companionship through all the tears and laughter during my journey at ISU.

Finally, I would like to express my heartfelt thanks to my family. My parents, my sister and my children have been my inspiration with their selfless love. My husband Mr. Chandrasekhar Singaraju has been my rock, with his unwavering confidence in my abilities and his boundless love, which has helped me fly.

S. S. 


\section{CONTENTS}

Page

ACKNOWLEDGMENTS

CONTENTS

ii

CHAPTER I TABLES

iv

CHAPTER II TABLES

$\mathrm{V}$

CHAPTER I FIGURES

vi

CHAPTER II FIGURES

vii

\section{CHAPTER}

I. BROAD SUBSTRATE SPECIFICITY OF PHOSPHOTRANSBUTYRYLASE FROM LISTERIA MONOCYTOGENES, A POTENTIAL PARTICIPANT IN AN ALTERNATIVE PATHWAY FOR PROVISION OF ACYL COA PRECURSORS FOR FATTY ACID BIOSYNTHESIS

ABSTRACT

1. Introduction

2. Materials and methods

2.1. Materials

2.2. Strains and plasmids

2.3. Purification of Ptb (EC 2.3.1.19)

2.4. Synthesis of acyl CoA substrates

2.5. Ptb assay

2.6. Steady state kinetic analysis

3. Results

3.1. Purification of Ptb 
3.2. Kinetic analysis of Ptb 12

3.3. Mechanism of action of Ptb 15

4. Discussion 16

4.1. Substrate specificity of Ptb 16

4.2. Ptb exhibits chain length specificity 18

4.3. Ptb demonstrates preference for branched-chain substrates 19

4.4. Ptb activity exhibits positive cooperativity with phosphate $\quad 20$

4.5. Ptb activity involves ternary complex formation 21

4.6. Conclusions 22

$\begin{array}{ll}\text { Acknowledgements } & 22\end{array}$

$\begin{array}{ll}\text { References } & 38\end{array}$

II. UTILIZATION OF MULTIPLE SUBSTRATES BY BUTYRATE KINASE FROM LISTERIA MONOCYTOGENES 42

ABSTRACT 43

1. Introduction 44

2. Materials and methods $\quad 47$

2.1. Materials

2.2. Cloning and expression of Buk $\quad 47$

2.3. Purification of Buk 48

2.4. Standardization of Buk assay 49

2.5. Kinetic analysis of Buk $\quad 50$

3. Results and discussion $\quad 51$

3.1. Buk demonstrates broad substrate specificity 51

3.2. Buk utilized unnatural BCCAs as its substrates 55

3.3. Buk prefers BCCAs at low temperatures 56

3.4. Buk catalysis occurs through a ternary complex
intermediate

4. Conclusions $\quad 58$

Acknowledgements $\quad 58$

$\begin{array}{ll}\text { References } & 68\end{array}$

$\begin{array}{lll}\text { III. } & \text { SUMMARY } & 72\end{array}$ 


\section{CHAPTER I TABLES}

Table $\quad$ Page

1. Steady State Kinetic Analysis Of Ptb From L. monocytogenes 24

2. Relative Activity Of Ptb 25 


\section{CHAPTER II TABLES}

Table $\quad$ Page

1. Steady State Kinetic Analysis Of Buk From L. monocytogenes At $37{ }^{\circ} \mathrm{C} \quad 59$

2. Steady State Kinetic Analysis Of Buk From L. monocytogenes At $10{ }^{\circ} \mathrm{C} \quad 60$ 


\section{CHAPTER I FIGURES}

Figure

Page

1. Structural Organization Of The bkd Operon Of L. monocytogenes 26

2. Reaction Catalyzed By Ptb

3. Proposed Alternative Pathway For Provision Of Acyl CoA Precursors For Fatty Acid Biosynthesis

4. Preparation Of Acyl CoA Substrates

5. SDS-PAGE Analysis Of Purified Preparation Of Ptb

6. Concentration-Dependence Of Straight-Chain Acyl CoA Substrates On Ptb Activity Assayed In The Acyl Phosphate Forming Direction

7. Concentration-Dependence Of Branched-Chain Acyl CoA Substrates On Ptb Activity Assayed In The Acyl Phosphate Forming Direction

8. Concentration-Dependence Of Butyryl Phosphate On Ptb Activity Assayed In The Acyl CoA Forming Direction

9. Comparison Of Catalytic Efficiency Of Ptb From L. monocytogenes 10403S With Different Substrates

10. Concentration Dependence Of Phosphate Utilization By LmPtb

11. Ptb Activity Follows A Sequential Kinetic Model

12. Mechanism Of Action Of Ptb 


\section{CHAPTER II FIGURES}

Figure

1. Analysis Of Purified LmBuk By SDS-PAGE 61

2. Reaction Catalyzed By LmBuk 62

3. Substrate Preference Of $L m B u k \quad 63$

3A. Concentration-Dependent Activity Of $L m B u k$ In The Presence Of SCCA Substrates At $37^{\circ} \mathrm{C}$

3B. Concentration-Dependent Activity Of $L m B u k$ In The Presence Of BCCA Substrates At $37^{\circ} \mathrm{C}$

3C. Concentration-Dependent Activity Of $\operatorname{LmBuk}$ At $10^{\circ} \mathrm{C}$ In The Presence Of SCCA And BCCA Substrates

4. Concentration Dependence Of ATP Utilization By LmBuk And Competitive Inhibition By ADP

5. LmBuk Catalysis Occurs Through A Ternary Complex Intermediate 
CHAPTER I

BROAD SUBSTRATE SPECIFICITY OF PHOSPHOTRANSBUTYRYLASE FROM LISTERIA MONOCYTOGENES, A POTENTIAL PARTICIPANT IN AN

ALTERNATIVE PATHWAY FOR PROVISION OF ACYL COA

PRECURSORS FOR FATTY ACID BIOSYNTHESIS

\section{Highlights}

- $\quad$ Ptb from L. monocytogenes exhibited broad substrate specificity

- Ptb preferred substrates with a chain length of 3 to 5 carbons

- An alkyl side chain was required for higher catalytic efficiency

- Ptb could utilize unnatural substrates such as 2-ethyl butyryl CoA

- Ptb catalysis involved ternary complex formation

\section{Keywords}

Phosphotransbutyrylase, Branched-chain carboxylic acids, Branched-chain fatty acids, Fatty acid biosynthesis, Membrane fatty acid composition, Acyl CoA 


\section{ABSTRACT}

Listeria monocytogenes, the causative organism of the serious food-borne disease listeriosis, has a membrane abundant in branched-chain fatty acids (BCFAs). BCFAs are normally biosynthesized from branched-chain amino acids via the activity of branched chain $\alpha$-keto acid dehydrogenase (Bkd), and disruption of this pathway results in reduced BCFA content in the membrane. Short branched-chain carboxylic acids (BCCAs) added as media supplements result in incorporation of BCFAs arising from the supplemented BCCA in the membrane of L. monocytogenes bkd mutant MOR401. High concentrations of the supplements also effect similar changes in the membrane of the wild type organism with intact $b k d$. Such carboxylic acids clearly act as fatty acid precursors, and there must be an alternative pathway resulting in the formation of their CoA thioester derivatives. Candidates for this are the enzymes phosphotransbutyrylase (Ptb) and butyrate kinase (Buk), the products of the first two genes of the $b k d$ operon. Ptb from $L$. monocytogenes exhibited broad substrate specificity, a strong preference for branched-chain substrates, a lack of activity with acetyl CoA and hexanoyl CoA, and strict chain length preference (C3-C5). Ptb catalysis involved ternary complex formation. Additionally, Ptb could utilize unnatural branched-chain substrates such as 2-ethyl butyryl CoA, albeit with lower efficiency consistent with a potential involvement of this enzyme in the conversion of the carboxylic acid additives into CoA primers for BCFA biosynthesis. 


\section{Introduction}

Listeria monocytogenes, the dangerous foodborne pathogen, grows actively at low temperatures and is a cause for concern due to the widespread use of refrigeration as a food preservation method [1]. Listeria outbreaks remain a continuing problem (http://www.cdc.gov/listeria/outbreaks). The economic costs of a 2008 L. monocytogenes outbreak in Canada linked to contaminated delicatessen meat from one processing plant, which resulted in 57 cases of listeriosis and 24 deaths, were estimated to be $\$ 242$ million Canadian dollars [2].

A fluid membrane ensured by a high content of branched-chain fatty acids (BCFAs), which have low phase transition temperatures, supports the growth of $L$. monocytogenes at low temperatures [3-5]. Branched-chain amino acids, namely isoleucine, leucine and valine through the activity of branched-chain amino transferase (Bcat) and branched-chain $\alpha$-keto acid dehydrogenase complex (Bkd) result in the formation of the CoA derivatives of 2- methyl butyrate, isobutyrate and isovalerate respectively, which are then elongated by the well-characterized dissociated fatty acid biosynthesis (FAS II) system to form membrane BCFAs [6]. Mutants of $L$. monocytogenes (cld-2/MOR401) lacking functional Bkd have low membrane BCFA content and demonstrate diminished growth at refrigeration temperatures, reduced tolerance to adverse environmental conditions such as oxidative stress, and poor survival in macrophages [7,8]. Growth of bkd mutants in Bacillus subtilis, L. monocytogenes and Staphylococcus aureus could be rescued by the addition of branched-chain carboxylic acids (BCCAs) to the medium [9-11]. 
Extensive studies on medium supplementation by Kaneda [12] in B. subtilis and by Sen et al. [13] in L. monocytogenes show that addition of a specific BCCA results in an increase in membrane fatty acids biosynthesized from that particular BCCA. The carboxylic acids that are capable of causing an alteration of membrane fatty acid composition are not restricted to the normal products of BCAA metabolism but also include unnatural C6 BCCAs that have a similar structure such as 2-ethyl butyrate, 2methyl pentanoate, and straight-chain carboxylic acids (SCCAs) such as butyrate and propionate $[12,13]$. Incorporation of novel fatty acids into the membrane as a result of elongation of unnatural C6 BCCAs is indicative of the entry of the supplements into the FAS II pathway as their respective acyl CoA derivatives since the only known source of membrane BCFAs is via biosynthesis [6]. Formation of acyl CoA derivatives despite the absence of functional Bkd confirms the presence of an alternate pathway which catalyzes the conversion of supplemented carboxylic acids into their respective acyl CoA end products [10,13]. Willecke and Pardee [11] first suggested the involvement of an alternate system in the conversion of BCCAs into precursors of fatty acids in a $b k d$ mutant of $B$. subtilis. Integration of the products of such a wide range of substrates into the membrane implies that the pathway involved in their activation must be remarkably flexible in its substrate specificity [10,13]. This pathway has not been characterized in $L$. monocytogenes thus far despite ample evidence of its existence.

Two genes, namely phosphotransbutyrylase $(p t b)$ and butyrate kinase $(b u k)$, have been identified upstream of the genes encoding the Bkd complex in the $b k d$ operon of $L$. monocytogenes based on sequence similarity to the bkd operon in B. subtilis and 
Enterococcus faecalis (Fig. 1) [7,14-16]. The molecular organization of these two genes in L. monocytogenes is markedly different from Clostridium acetobutylicum in which ptb and $b u k$ form a separate operon expressed from a single promoter [17]. Buk (EC 2.7.2.7) is known to phosphorylate short chain carboxylic acids in the fermentative organism Clostridium acetobutylicum and a marine spirochete MA-2 [18,19]. Ptb (EC 2.3.1.19) from C. acetobutylicum is an enzyme catalyzing the reversible conversion of acyl phosphates to acyl CoAs [20] (Fig. 2). We propose that these two enzymes, given their functions and location in the $b k d$ operon, reverse their physiological direction in the presence of exogenous carboxylic acids and catalyze the conversion of these substrates into their corresponding acyl CoA thioesters (Fig. 3).

Ptb has been well studied in C. acetobutylicum, an important producer of industrial solvents, and it plays a crucial role in the energy metabolism in the acidogenesis phase leading to the production of ATP by substrate phosphorylation [2022]. Similar studies in E. faecalis demonstrate the involvement of Ptb in branched-chain amino acid catabolism resulting in energy production and secretion of catabolic end products such as 2-methyl butyrate and isovalerate [16]. These studies on Ptb were focused on its involvement in energy metabolism and have been limited in scope.

Ptb from L. monocytogenes is a putative 288 amino acid protein with $38.5 \%$ identity to the enzyme from $C$. acetobutylicum and $41 \%$ identity to Ptb from E. faecalis. In this work we describe the purification of Ptb from L. monocytogenes expressed in E. coli and investigation of its substrate preferences and enzyme kinetic constants $\left(K_{M}\right.$ and $\left.k_{c a t}\right)$. The results indicate that Ptb demonstrates broad substrate specificity, which is 
consistent with our hypothesis of its role in the incorporation of exogenous carboxylic acids into the membrane fatty acids. SCCAs such as propionate and butyrate, which are used as food preservatives, additionally may contribute to the control of $L$.

monocytogenes by resulting in the biosynthesis of straight-chain fatty acids that rigidify the membrane $[10,13]$. The substantial activity with these substrates suggests that Ptb may play a role in this process.

\section{Materials and methods}

\subsection{Materials}

Pentanoyl CoA was purchased from Crystalchem (Downers Grove, IL). All other materials used in this work including the chemicals utilized for synthesis of 2-methyl butyryl CoA, 2-ethyl butyryl CoA and 2-methyl pentanoyl CoA were purchased from Sigma Aldrich (St. Louis, MO).

\subsection{Strains and plasmids}

L. monocytogenes $10403 \mathrm{~S}$ was grown in Brain Heart Infusion (BHI) broth (Becton Dickinson, Sparks, MD) and used for isolation of genomic DNA using a Masterpure genomic DNA purification kit according to the manufacturer's instructions (Epicenter, Madison, WI). Primers (forward 5'

GGGGAGGTCGACAAATGACAAAAAGCAGA TTTTTTTCA and reverse 5'GGGGAGCTCGAGTTTCTCAACTAGTCTTACAG) were designed with restriction sites for cloning and used for amplification of ptb from L. monocytogenes $10403 \mathrm{~S}$ genomic DNA. The amplified gene (864 bp) was ligated into the expression vector pET28a (Novagen, Madison, WI) using T4 DNA ligase (Fermentas, Waltham, MA) to 
generate pET28a-ptb and transformed into competent Escherichia coli BL21 (DE3) cells. Kanamycin $(50 \mu \mathrm{g} / \mathrm{ml})$ was used as the selection agent for growth of pET28a and pET28a-ptb carrying E. coli cells in Luria broth (Becton Dickinson, Sparks, MD). Purification of DNA, ligation and transformation were performed according to the manufacturer's instructions (Qiagen, Valencia, CA).

\subsection{Purification of Ptb (EC 2.3.1.19)}

An overnight culture of $E$. coli BL21 DE3 cells carrying pET28a-ptb was used to inoculate $500 \mathrm{ml}$ Luria broth ( $2 \%$ inoculum) which was incubated at $37{ }^{\circ} \mathrm{C}$ until the $\mathrm{OD}_{600}$ reached 0.6 . Overexpression of Ptb was induced by the addition of isopropyl $\beta$ - Dthiogalactopyranoside (IPTG) to a final concentration of $1 \mathrm{mM}$. The cells were harvested by centrifugation at $4{ }^{\circ} \mathrm{C}$ at $3,000 \mathrm{~g}$ and the pellets were stored at $-80^{\circ} \mathrm{C}$. To purify Ptb, the cell pellet was resuspended in binding buffer $(1.5 \mathrm{M}$ sodium chloride- $\mathrm{NaCl}, 25 \mathrm{mM}$ $\mathrm{N}$-(2-Hydroxyethyl) piperazine-N'-(2-ethanesulfonic acid) [HEPES], $1 \mathrm{mM} \mathrm{MgCl} 2,5$ $\mathrm{mM}$ imidazole, $5 \%$ glycerol and $2 \mathrm{mM} \beta$-mercapto ethanol $\mathrm{pH} 7.5$ ) and the cells were broken using a French press at 16,000 psi with two passes through the machine. Debris was removed by centrifugation at 20,000 $\mathrm{g}$ for $30 \mathrm{~min}$ and the cell-free extract was allowed to bind for one hour with nickel-chelated nitrilotriacetic acid $\left(\mathrm{Ni}^{2+}-\mathrm{NTA}\right)$ resin (Thermo Scientific, Waltham, MA), which was pretreated with binding buffer. The resin was then packed into a column and washed with 5 column volumes of wash buffer (1.5 $\mathrm{M} \mathrm{NaCl}, 25 \mathrm{mM}$ HEPES, $1 \mathrm{mM} \mathrm{MgCl} 2,10 \mathrm{mM}$ imidazole, $5 \%$ glycerol and $2 \mathrm{mM} \beta$ mercaptoethanol, $\mathrm{pH}$ 7.5). The bound hexa histidine-tagged Ptb was then eluted with $4 \mathrm{ml}$ of elution buffer (1.5 M NaCl, $25 \mathrm{mM}$ HEPES, $1 \mathrm{mM} \mathrm{MgCl} 2,250 \mathrm{mM}$ imidazole, $25 \%$ 
glycerol and $2 \mathrm{mM} \beta$-mercaptoethanol, $\mathrm{pH}$ 7.5). The concentration of protein was determined by the Bradford assay using bovine serum albumin as the standard (Biorad, Hercules, CA).

\subsection{Synthesis of acyl CoA substrates}

The acyl CoA substrates 2-methyl butyryl CoA, 2-ethyl butyryl CoA were prepared (Fig. 4) according to the protocol outlined by Stadtman [23]. Briefly, the mixed anhydride was prepared by the addition of $0.1 \mathrm{~mol}$ of the organic acid and anhydrous pyridine each to $10 \mathrm{ml}$ ice-cold ethyl ether drop wise. This was followed by the addition of $0.1 \mathrm{~mol}$ of ethyl chloroformate drop wise to the mixture. The mixture was stirred for one hour in an ice bath. The insoluble pyridine hydrochloride that formed as a precipitate was removed by filtration and discarded. Next an aqueous solution of coenzyme A was prepared in $0.2 \mathrm{M} \mathrm{KH}_{2} \mathrm{CO}_{3}$ at $\mathrm{pH}$ 7.5. To this solution an equimolar amount of the mixed anhydride was added dropwise with constant stirring on ice for five minutes. The solution was tested for the presence of free CoA by the nitroprusside test as described by Toennies and Kolb [24]. Additional mixed anhydride was added until the nitroprusside test no longer revealed free $\mathrm{CoA}$. The $\mathrm{pH}$ was then adjusted to 6.0 by the addition of $1 \mathrm{M} \mathrm{HCl}$ and the aqueous layer was extracted with an equal volume of ethyl ether three times to remove unreacted mixed anhydride. The remaining traces of ethyl ether were removed by bubbling nitrogen through the solution. The prepared acyl CoA substrate was quantitated by the hydroxylamine method [25]. 


\subsection{Ptb assay}

All kinetic studies were performed at room temperature $\left(23-25^{\circ} \mathrm{C}\right)$ in a reaction volume of $200 \mu \mathrm{l}$ in microcuvettes (path length $=1 \mathrm{~cm}$ ). Ptb was assayed in the acyl phosphate forming direction according to the method of Klotsch [26] as described by Weisenborn et al., [20]. Ptb catalyzes the nucleophilic attack from inorganic phosphate on an existing acyl-CoA derivative to produce the corresponding acylphosphate derivative and concomitantly liberates coenzyme-A (Fig. 2, step 1). The free sulfhydryl group on coenzyme-A was then quantified by reacting it with Ellman's reagent [5,5'dithiobis-(2-nitrobenzoic acid), (DTNB)] [27]. Free thiols react with the disulfide bond in DTNB and liberate 5-thio-2-nitrobenzoic acid (TNB), which at neutral and alkaline pH ionizes to produce a yellow color which we measured spectrophotometrically $(412 \mathrm{~nm}$, Nanodrop 2000c UV-Vis spectrophotometer, Wilmington, DE) (Fig. 2, step 2). The production of TNB is stoichiometric with the amount of thiol present and thus was used calculate the initial velocity of Ptb production of each acylphosphate derivative.

Ptb assay in the reverse direction was performed by the colorimetric measurement of the released phosphate according to the method described by Helms et al. [28] based on the scheme outlined earlier [29]. The assay solution $(200 \mu \mathrm{l})$ contained $100 \mathrm{mM}$ Tris (pH 7.5), $500 \mu \mathrm{M}$ CoA and variable butyryl phosphate $(200 \mu \mathrm{M}-3 \mathrm{mM})$ and the reaction was initiated by the addition of Ptb. The reaction was incubated at $25^{\circ} \mathrm{C}$ for 10 minutes and $50 \mu \mathrm{l}$ of the assay solution was transferred into a 96-well microplate on ice. The reaction was stopped by addition of $80 \mu \mathrm{l}$ of freshly prepared ice-cold acidic stop solution (0.5 M HCl, $0.129 \mathrm{~g}$ ascorbic acid and $0.2 \mathrm{ml}$ of freshly prepared $10 \%$ ammonium 
molybdate solution in a total volume of $4.2 \mathrm{ml}$ ). After incubation on ice for 10 minutes, $120 \mu \mathrm{l}$ of ACG solution (150 mM sodium m-arsenite, $70 \mathrm{mM}$ sodium citrate, $350 \mathrm{mM}$ acetic acid) was added and incubated for 5 minutes at $37^{\circ} \mathrm{C}$. The color formed was estimated at $800 \mathrm{~nm}$. The experimental data were plotted as a function of butyryl phosphate concentration and the kinetic constants were determined by curve fitting of the data to Michaelis-Menten equation.

\subsection{Steady state kinetic analysis}

The assay for Ptb activity was standardized by using butyryl CoA as the substrate with a saturating concentration of inorganic phosphate $(0.1 \mathrm{M})$ in the assay mixture. Enzyme-dependent rate of product formation in terms of the change in absorbance at 412 $\mathrm{nm}$ was studied and it was decided that a final concentration of Ptb in the assay of 5-10 $\mathrm{nM}$ would be used to obtain reaction velocities. Assay medium contained: $50 \mathrm{mM}$ Tris (pH 7.5), $4 \mathrm{mM} \mathrm{MgCl}, 0.08 \mathrm{mM}$ DTNB, $0.1 \mathrm{M} \mathrm{KH}_{2} \mathrm{PO}_{4}$ and varying amounts of acyl CoA $(10 \mu \mathrm{M}$ to $500 \mu \mathrm{M})$. Stock solutions of the acyl CoA substrates $(10 \mathrm{mM})$ were prepared using $50 \mathrm{mM}$ Tris- $\mathrm{HCl}(\mathrm{pH} 4.0)$ and the concentrations were calculated based on the purity of the substrate as reported by the manufacturer. Tris buffer at low $\mathrm{pH}$ was chosen for preparing stock solutions in order to rapidly bring up the $\mathrm{pH}$ of the working solution prior to use. Each substrate was diluted to $1 \mathrm{mM}$ concentration prior to use with $50 \mathrm{mM}$ Tris- $\mathrm{HCl}(\mathrm{pH} 7.5)$. Reactions were initiated by the addition of Ptb and absorbance $(412 \mathrm{~nm})$ was recorded at two second intervals for the duration of the reaction $(60 \mathrm{sec})$. Initial rates were calculated from the linear portion of the reaction curve using Microsoft Excel and plotted against the substrate concentration, and apparent kinetic parameters 
were determined using KaleidaGraph software by fitting the data to the MichaelisMenten equation, $v=\mathrm{Vmax} *[\mathrm{~S}] /\left(K_{M}+[\mathrm{S}]\right)$ where $\mathrm{Vmax}$ is the maximum velocity and $K_{M}$ is the substrate concentration that produces half maximal velocity and [S] is the substrate concentration (Kaleidagraph, Synergy software, Reading, PA). At least three replicates of each reaction were performed and the results were presented as the mean \pm standard error of the mean. Coenzyme-A (25-125 $\mu \mathrm{M})$ (Sigma-Aldrich, St. Louis, MO) was used to establish a standard curve. Assays were conducted in the absence of enzyme to determine background degradation of the substrate, if any, and also in the absence of the substrate to rule out interference of $\beta$-mercaptoethanol. The apparent $K_{M}$ and $k_{c a t}$ values were determined for a variety of straight-chain and branched-chain acyl CoA compounds listed in Table 1. Ptb was assayed in the presence of excess isovaleryl CoA $(200 \mu \mathrm{M})$ and varying inorganic phosphate concentrations $(50 \mu \mathrm{M}-25 \mathrm{mM})$ in order to determine the kinetic constants with respect to inorganic phosphate.

\section{Results}

\subsection{Purification of Ptb}

Ptb was purified by its $\mathrm{N}$-terminal His-tag through $\mathrm{Ni}^{2+}-\mathrm{NTA}$ affinity purification as described in Methods. Loss of catalytic activity due to precipitation of purified Ptb was observed at concentrations ranging from $1-6 \mathrm{mg} / \mathrm{ml}$ in $50 \mathrm{mM}$ Tris $(\mathrm{pH} 7.5)$ and in 25 mM HEPES (pH 7.5) buffers. Similar problems with stabilization of purified Ptb from Clostridium beijerinckii have been reported by Thompson and Chen [30], which were resolved by storage in high $\mathrm{NaCl}$ concentrations (1.3-3M). Ptb from L. monocytogenes could be stored for two weeks without detectable loss of activity by dilution of eluted 
protein to $<0.5 \mathrm{mg} / \mathrm{ml}$ in HEPES buffer with $1.5 \mathrm{M} \mathrm{NaCl}$. Sodium dodecyl sulfatepolyacrylamide gel electrophoresis (SDS-PAGE) analysis confirmed the calculated molecular mass of Ptb ( $34 \mathrm{kDa})$ and its purity (Fig. 5).

\subsection{Kinetic analysis of Ptb}

\subsubsection{Straight chain acyl CoA substrates}

It was of interest to understand the substrate specificity of Ptb in order to determine if it could fulfill the role of activation of different carboxylic acids. To this end Ptb was assayed systematically with acyl CoA substrates differing in carbon chain length by one carbon (C2-C6). The data were fitted to the Michaelis -Menten equation (Fig. 6) and the kinetic constants presented in Table 1. As shown in Table 1, the $K_{M}$ of butyrate was $79.9 \mu \mathrm{M}$ and was the lowest of all the SCCAs tested. This $K_{M}$ value was similar to those previously reported for Ptb from other organisms [20,31]. The shortest and longest substrates tested, acetyl CoA (C2) and hexanoyl CoA (C6), proved to be poor substrates for Ptb judging by their poor fit to the Michaelis-Menten equation and low catalytic efficiency $\left(k_{c a t} / K_{M}\right)$ suggesting that the carbon chain length was suboptimal for efficient binding to the active site. The overall catalytic efficiency and the turnover number $\left(k_{c a t}\right)$ for this series of substrates suggested that increase/decrease in chain length from the optimal size of $\mathrm{C} 4$ (butyryl CoA-8.43 $\mu \mathrm{M}^{-1} \mathrm{sec}^{-1}$ ) resulted in a decrease in catalytic efficiency with a sharp drop with an increase/decrease of two carbons (C2-0.3 $\mu \mathrm{M}^{-1} \mathrm{sec}^{-}$ ${ }^{1}$ and C6- $1.7 \mu \mathrm{M}^{-1} \mathrm{sec}^{-1}$ ) (Fig. 8). From these data, we concluded that the ideal chain length of substrates for Ptb was C3-C5. 
Ptb was also assayed in the acyl CoA forming direction using butyryl phosphate. The $K_{M}$ for butyryl phosphate was found to be $1.04 \mathrm{mM}$, which is higher than the previously reported value for $K_{M}$ for butyryl phosphate, and a corresponding catalytic efficiency ( $k_{c a t} / K_{M)}$ of 1.05 (Fig. 8) [20]. Additionally, presence of butyryl phosphate did not exhibit significant inhibition in the acyl phosphate forming direction (data not shown).

\subsubsection{Branched-chain acyl CoA substrates}

In order to understand the substrate preferences of Ptb in detail we also examined its activity with a variety of branched-chain substrates. Our measurements were limited by the commercial availability of branched-chain substrates (isobutyryl CoA and isovaleryl CoA) and the substrates that we could prepare (2-methyl butyryl CoA and 2ethyl butyryl CoA). Nonetheless, our studies utilized enough substrate structural diversity to deduce critical features of the Ptb active site (Fig. 7). Presence of an alkyl side chain improved the binding affinity of the substrate to Ptb as evidenced by the $K_{M}$ values (Table 1). Additionally, the $K_{M}$ values of the different branched-chain substrates were similar suggesting that the presence of the alkyl side chain likely produces critical contacts within the active site resulting in improved binding. Perhaps the presence of the side chain restricted freedom of movement of the substrate in the active site, and thus resulted in increased turnover numbers $\left(k_{c a t}\right)$ of the branched-chain substrates in comparison to straight-chain substrates of the same chain length (e.g. butyryl-CoA-673.9 $\sec ^{-1}$, isovaleryl-CoA $1043.4 \mathrm{sec}^{-1}$, 2-methyl butyryl-CoA-849.1 $\mathrm{sec}^{-1}$ ) (Table 1). Furthermore, binding affinity did not appear to be influenced by the branch position nor 
by the length of the side chain as demonstrated by the similar $K_{M}$ values for isovalerylCoA ( $\beta$-position) and 2-methylbutyryl-CoA ( $\alpha$-position) and 2-ethylbutyryl-CoA (ethyl branch in the $\alpha$-position) (Table 1). However, the increase in side chain length was found to be associated with lower $k_{c a t}$ values suggesting a decrease in product formation.

Analysis of our experimental data (Fig. 9, Table $1 \& 2$ ) indicated a strong preference for substrates with an alkyl side chain.

\subsubsection{Inorganic phosphate}

The kinetic constants of Ptb with respect to inorganic phosphate were determined at excess isobutyryl CoA, isovaleryl-CoA and propionyl CoA concentrations ( $200 \mu \mathrm{M})$ based on curve fitting to the Hill equation (Fig. 10a and 10b). Others have reported that high concentrations of inorganic phosphate can inhibit Ptb from some other bacteria [30]. It has also been reported that $K_{M}$ values for phosphate can differ with various acyl-CoA substrates [30]. Consequently, we examined the phosphate parameters more closely in $L$. monocytogenes Ptb. We did not observe any inhibition by increasing concentrations of inorganic phosphate in our experiments.

When the specific activity of Ptb was plotted as a function of phosphate concentration (Fig. 10a. and 10b), product formation was minimal at low phosphate concentrations and an increase in activity was observed with concurrent increase in phosphate concentration $(>1 \mathrm{mM})$ until a maximum was obtained at about $15-20 \mathrm{mM}$ of phosphate. The sigmoidal nature of the curve was readily apparent only at low concentrations of phosphate, thus confirming positive cooperativity, and curve fitting of the data to the Hill equation $\left(v=V \max *[S]^{\mathrm{n}} / \mathrm{K}+[\mathrm{S}]^{\mathrm{n}}\right.$, where $\mathrm{n}$ is the Hill coefficient $)$ 
allowed the estimation of the parameters for binding of phosphate to Ptb (Fig. 10a. and 10b). The apparent $K_{M}$ for phosphate when isobutyryl CoA was utilized as the cosubstrate was found to be $5.35 \pm 0.55 \mathrm{mM}$ with a Hill coefficient of $2.11 \pm 0.37$ and comparable parameters were obtained with isovaleryl CoA $\left(K_{M}-2.78 \mathrm{mM}\right)$ as the second substrate. However, the apparent $K_{M}$ was found to be higher when the second substrate utilized was not a preferred substrate, as in the case of propionyl CoA $\left(K_{M}=16.4 \mathrm{mM}\right)$, a $\mathrm{C} 3$ compound which lacks a methyl branch unlike isobutyryl CoA and isovaleryl CoA.

\subsection{Mechanism of action of Ptb}

The initial rates of Ptb were further analyzed at varying concentrations of isovaleryl $\mathrm{CoA}$ in the presence of different fixed concentrations of inorganic phosphate $(10,25$ and $100 \mathrm{mM})$. These data were used to determine the binding mechanism of Ptb which utilized the two substrates, acyl CoA and inorganic phosphate, with the formation of two products, CoA and acyl phosphate. Experiments were conducted to determine whether Ptb catalysis followed the ping-pong model or required ternary complex formation. Data were normalized to the $k_{\text {cat }}$ of isovaleryl CoA at $100 \mathrm{mM}$ inorganic phosphate and fitted to a double reciprocal plot (Fig. 11). The double reciprocal fit of the data yielded lines intersecting at a single point on the $\mathrm{Y}$-axis and was consistent with ternary complex formation rather than a ping-pong mechanism. The intersection of the lines at a single point on the $\mathrm{Y}$ - axis confirmed the mechanism to be that of rapid equilibrium ordered mechanism as outlined schematically in Fig. 12 [32]). Furthermore, Scheme 1 describing a simple ternary complex mechanism of action was mathematically simplified to the expression in equation 1 where $\mathrm{x}$ is the concentration of the varying 
substrate (isobutyryl CoA) and $\mathrm{S}$ is the concentration of the fixed substrate (inorganic phosphate). The initial rate data were fitted globally to equation 1 using the graphing software OriginPro (OriginLab, Northampton, MA) which confirmed the mechanism of action to involve ternary complex formation.

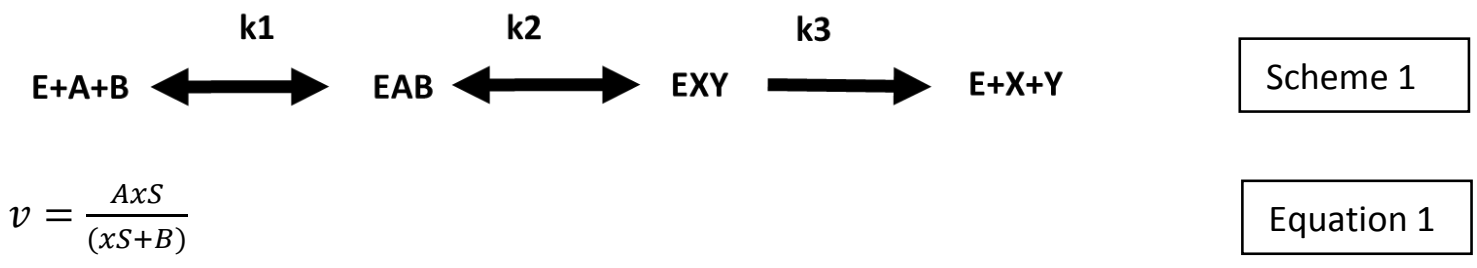

\section{Discussion}

\subsection{Substrate specificity of Ptb}

The high membrane BCFA content of L. monocytogenes $10403 \mathrm{~S}$ is ensured due to the strong affinity of its FabH enzyme towards the precursors of BCFAs [33]. Although the membrane fatty acid composition of the wild-type organism is affected only by high external concentrations of propionate, butyrate and certain unnatural BCCAs, the $b k d$ mutant MOR401(cld-2) is more susceptible to manipulation by supplementation of the media by various carboxylic acids $[10,13]$. Sen et al. [13] show that in addition to the natural products of amino acid catabolism, certain BCCAs with similar structural characteristics such as 2-ethyl butyrate and 2-methyl pentanoate also cause significant alteration of the membrane fatty acid composition to reflect the incorporation of the fatty acids resulting from the elongation of the added BCCAs. These studies strongly indicate the presence of a bypass pathway forming CoA thioesters of the BCCAs and SCCAs for entry into the bacterial FAS II system. Ptb and Buk, whose activities resemble that of 
phosphotransacetylase ( $p t a)$ and acetate kinase ( $a c k$ ) respectively, were concluded to be the most likely candidates due to their reported ability to utilize multiple substrates in other organisms, reversibility of their catalytic activity, and their location in the $b k d$ operon $[18,20,34,35]$.

Our investigation into the kinetic characteristics of Ptb revealed its substantial activity with a wide range of acyl CoA substrates thus supporting our hypothesis. The substrates that were studied differed in their chain length and also in the presence and location of alkyl side chains. The broad substrate specificity of the enzyme is in agreement with the available data on Ptb from C. acetobutylicum and Ptb from E. faecalis $[16,20]$. Although Ptb has been studied in these organisms, the scope of these investigations has been limited to exploration of its activity with respect to the secreted products, such as butyrate in case of $C$. acetobutylicum, and the end products of BCAA catabolism in E. faecalis, with the inclusion of a few closely related compounds [16,20,22]. L. monocytogenes does not secrete these products under either aerobic or anaerobic growth conditions in defined medium according to a report by Romick et al. [36]. Additionally, a relationship between the activities of the $b k d$ operon and membrane fatty acid composition could not be established in E. faecalis although $21 \%$ of the membrane fatty acids were BCFAs [16]. In contrast, Ptb from L. monocytogenes demonstrates considerable activity with the various substrates which have been previously demonstrated to influence the membrane fatty acid composition thus making it a likely candidate in the activation of exogenous carboxylic acids. Such a relationship between the activity of Ptb and the membrane composition has not been reported thus far. 


\subsection{Ptb exhibits chain length specificity}

Although Ptb demonstrated broad substrate specificity, the activity is limited to substrates having a carbon chain length of C3-C5. Negligible activity with acetyl CoA as the substrate indicates that Ptb is distinct from Pta and that it is not involved in acetyl CoA metabolism. These data agree with previously published reports on Ptb from $C$. acetobutylicum, which likewise demonstrated no activity with acetyl CoA as the substrate, and the fact that addition of acetate into the medium does not cause any change in the membrane fatty acid composition $[10,20,22]$. Long chain fatty acids such as C14:0, C16:0 and C18:1 and addition of monolaurin and bile salts to the medium cause alteration of the membrane fatty acids to reflect the added substrates in L. monocytogenes [37-39]. While such fatty acids are incorporated into the membrane, their activation by Ptb is unlikely since a drastic drop in catalytic efficiency was exhibited by Ptb with hexanoyl CoA (C6) as its substrate when compared with its activity with pentanoyl CoA (C5) (Fig. $6 \& 8$, Table $1 \& 2$ ). Recent reports by Parsons et al. [40,41] revealed a novel pathway involving the activation of long chain fatty acids by means of phosphorylation by a fatty acid kinase (Fak) thereby resulting in the production of fatty acyl phosphate. This pathway appears to be a more likely mechanism for activation of long chain fatty acids.

It is noteworthy that Ptb exhibited the highest affinity towards butyryl CoA among the straight chain substrates. SCCAs, mainly acetate, propionate and butyrate, make up the majority of the byproducts of anaerobic metabolism by the mammalian gut commensal bacteria and L. monocytogenes is exposed to these carboxylic acids during infection [42]. Exposure to butyrate results in reduced expression of virulence genes in $L$. 
monocytogenes and Salmonella enterica Enteriditis thus indicating its role of a signal molecule $[43,44]$. The role of butyrate as a signal molecule is also apparent in Enterohemorrhagic E. coli (EHEC) in which it causes an upregulation of the virulence genes and increased motility [45]. Moreover, increased expression of solvent producing and stationary phase genes coinciding with a butyryl phosphate peak has been reported in C. acetobutylicum. Butyryl phosphate is hypothesized to behave as a small molecule phosphate donor similar to acetyl phosphate in E. coli in its role as a global gene regulator in C. acetobutylicum [46]. Additionally, high concentrations of butyrate result in growth inhibition due to incorporation of SCFAs in the membrane of $L$. monocytogenes, a property that could be used to aid in control of the organism $[10,13]$. Butyrate has been used as a feed additive to aid in the control S. enterica Enteriditis in turkeys and chicken $[47,48]$.

\subsection{Ptb demonstrates preference for branched-chain substrates}

Ptb from L. monocytogenes preferred branched-chain CoA substrates, especially the natural degradation products of the branched-chain amino acids. The order of catalytic efficiency of Ptb in decreasing order was isobutyryl CoA $>$ isovaleryl CoA $>2$ methyl butyryl CoA > 2-ethyl butyryl CoA (Table 1). Such distinct preference of Ptb towards branched-chain substrates has not been established in any organism thus far. Ward et al. [16] showed that Ptb was involved in the catabolism of branched-chain amino acids by determining its activity with various substrates compared to that of butyrate. However, the kinetic constants were not determined for the other related acyl CoA substrates. FabH has been reported to prefer BCFA substrates in the order 2-methyl 
butyryl $\mathrm{CoA}>$ isovaleryl $\mathrm{CoA}>$ isobutyryl $\mathrm{CoA}$ at $30{ }^{\circ} \mathrm{C}$ in L. monocytogenes [33]. The substrate specificity of Ptb is unlike that of Bkd, the normal source of the intracellular acyl CoA pool, whose preference is $\alpha$-keto methyl valerate $>\alpha$-keto isovalerate $>\alpha$-keto isocaproate and results in the formation of 2-methyl butyryl CoA, isobutyryl CoA and isovaleryl CoA respectively in $B$. subtilis [6]. This variation in the substrate specificities of the two enzymes may help explain the lack of restoration of the membrane fatty acid composition in the bkd mutant in L. monocytogenes (cld-2), to that of the wild type organism, despite the presence of equal amounts of the three BCCAs [7]. In other words, the significant branched-chain acyl CoA product formation by Ptb due to exogenous supplementation could outcompete the endogenous substrates for FabH and the substrate preference of Ptb could be reflected in the membrane fatty acid composition.

\subsection{Ptb activity exhibits positive cooperativity with phosphate}

Ptb from L. monocytogenes displays positive cooperativity with respect to phosphate in the absence of limiting conditions of acyl CoA substrate and is also not inhibited by increasing concentrations of phosphate. Wiesenborn et al. [20] investigated the binding of phosphate by Ptb from C. acetobutylicum and reported non-competitive inhibition at high concentrations of phosphate in the presence of excess butyryl CoA. Similar studies by Thompson and Chen [30] on Ptb from C. beijerinckii showed higher $K_{M}$ values for phosphate when the cosubstrate was changed from butyryl CoA to a weaker substrate namely acetoacetyl CoA. Both the studies utilized a minimum concentration of $5 \mathrm{mM}$ for phosphate for their kinetic investigations, which precluded a demonstration of positive cooperativity by the enzyme. We investigated Ptb from $L$. 
monocytogenes using a minimum concentration of $50 \mu \mathrm{M}$ to a maximum of $25 \mathrm{mM}$ which allowed the observation of sigmoidal behavior by the enzyme, a confirmation of positive cooperativity. Although binding of phosphate molecules at more than one site was offered as an explanation for the nonlinearity of the double reciprocal plots by Wiesenborn et al. [20], our studies suggest that binding of the acyl CoA molecule likely influences the binding of phosphate within the active site. Thus binding of an optimal substrate, such as isobutyryl CoA, was accompanied by a lower $K_{M}$ for phosphate, indicating higher affinity, whereas binding of a substrate that is suboptimal, such as propionyl CoA, resulted in a higher $K_{M}$ for phosphate.

\subsection{Ptb activity involves ternary complex formation}

The mechanism of action of Ptb was confirmed to be that of rapid equilibrium ordered mechanism (Fig. 11) by the global fit of the initial rate data to the mathematical expression corresponding to the ternary complex formation or random $\mathrm{Bi} \mathrm{Bi}$ binding scheme (Equation 1). This was similar to the mechanism determined for Ptb in $C$. acetobutylicum in which the data fit to a family of parabolic curves but was still not consistent with the fit for the ping pong mechanism. Formation of the ternary complex in phosphate acetyltransferase (pta) has been demonstrated in Clostridium kluyveri and also in the thermophilic bacterium Methanosarcina thermophila $[34,49,50]$. However, the double reciprocal plot of the data in $M$. thermophila shows lines intersecting to the left of the Y-axis and is unlike that of Ptb which shows the lines intersecting on a single point on the $\mathrm{Y}$ - axis [49]. Convergence of the lines to a point on the $\mathrm{Y}$ axis suggests the mechanism of action to be that of random $\mathrm{Bi} \mathrm{Bi}$ and rapid equilibrium ordered 
mechanism wherein the enzyme forms a complex with both the substrates before the formation of products by chemical alteration [32]. This is the first report of the mechanism of action of Ptb from L. monocytogenes.

\subsection{Conclusions}

Analysis of Ptb from L. monocytogenes reveals that it is active with a large number of substrates and prefers substrates with a carbon chain length of C3-C5 and the presence of an alkyl side chain. Activity with natural SCCAs and BCCAs and also with unnatural substrates such as 2-ethyl butyrate, that are capable of altering the membrane fatty acid composition, strongly indicates that Ptb is the probable candidate for the alternate pathway for primer production for fatty acid biosynthesis. Butyrate is likely involved in regulation of gene expression since exposure to butyrate leads to decreased expression of virulence genes in L. monocytogenes. Additionally, high concentrations of butyrate also lead to diminished growth of L. monocytogenes suggesting a possible control strategy for the organism. The high activity of Ptb with butyryl CoA indicates that it could be involved in signal processing upon exposure of L. monocytogenes to butyrate.

\section{Acknowledgements}

This work was supported by grant 1 R15 AI099977-01 from the National Institutes of Health to Brian J Wilkinson and Craig Gatto and 1 R15 GM61583 to Craig Gatto. This work was also supported by the R. D. Weigel grant from the Beta Lambda Chapter of the Phi Sigma Biological Honor Society at Illinois State University. The funding sources had no role in study design, collection, analysis and interpretation of data, writing of this manuscript, or the decision to submit it for publication. 
http://www.sciencedirect.com/science/article/pii/S138819811630155X 


\section{Table 1}

Steady state kinetic analysis of Ptb from L. monocytogenes

\begin{tabular}{lccc}
\hline Substrate & $k_{\text {cat }}\left(\mathrm{sec}^{-1}\right)$ & $K_{M}(\mu \mathrm{M})$ & $\begin{array}{c}k_{c a t} / K_{M} \\
\left(\mu \mathrm{M}^{-1} \mathrm{sec}^{-1}\right)\end{array}$ \\
\hline Straight-chain & & & \\
Acetyl CoA & $302.4 \pm 103.9$ & $992.1 \pm 471.9$ & 0.3 \\
Propionyl CoA & $518.8 \pm 25.8$ & $190.6 \pm 20.9$ & 2.72 \\
Butyryl CoA & $673.9 \pm 25.2$ & $79.9 \pm 8.8$ & 8.43 \\
Pentanoyl CoA & $676.1 \pm 19$ & $160.8 \pm 10.5$ & 4.2 \\
Hexanoyl CoA & $47 \pm 2.6$ & $31.5 \pm 8.4$ & 1.7 \\
& & & \\
Branched-chain & & & 21.4 \\
Iso butyryl CoA & $1263.4 \pm 31.7$ & $57.9 \pm 4.8$ & 19.6 \\
Isovaleryl CoA & $1043.4 \pm 12.2$ & $53.2 \pm 2.1$ & 17.7 \\
2-methylbutyryl CoA & $849.1 \pm 18$ & $48.1 \pm 3.4$ & 2.3 \\
2-ethyl butyryl CoA & $100.9 \pm 11$ & $43.8 \pm 20.3$ & \\
\hline
\end{tabular}

Initial velocities were measured at $25^{\circ} \mathrm{C}$ in the presence of $100 \mathrm{mM}$ phosphate at $\mathrm{pH}$ 7.5. The $k_{c a t}$ and $K_{M}$ were calculated from the least squares fit of the experimental data from the different substrates to the Michaelis-Menten equation. The values indicated are the mean of experiments performed at least in triplicate \pm SEM. 
Table 2

Relative activity of Ptb

\begin{tabular}{lc}
\hline Substrate & Relative activity \% \\
\hline Isobutyryl CoA & 100.0 \\
Isovaleryl CoA & 86.7 \\
2-methyl butyryl CoA & 73.8 \\
Butyryl CoA & 47.1 \\
Pentanoyl CoA & 32.6 \\
Propionyl CoA & 22.3 \\
2-ethyl butyryl CoA & 7.1 \\
Hexanoyl CoA & 4.8 \\
Acetyl CoA & 4.2 \\
\hline
\end{tabular}

Relative activity was calculated from the experimental data obtained at $100 \mu \mathrm{M}$ acyl CoA concentration and $100 \mathrm{mM}$ phosphate measured at $25^{\circ} \mathrm{C}$ under the same buffer conditions as stated in "Materials and Methods". The activity of isobutyryl CoA $\left(k_{c a t}\right)$ was designated as $100 \%$ and the relative activity of the other substrates were calculated in comparison to isobutyryl CoA. 


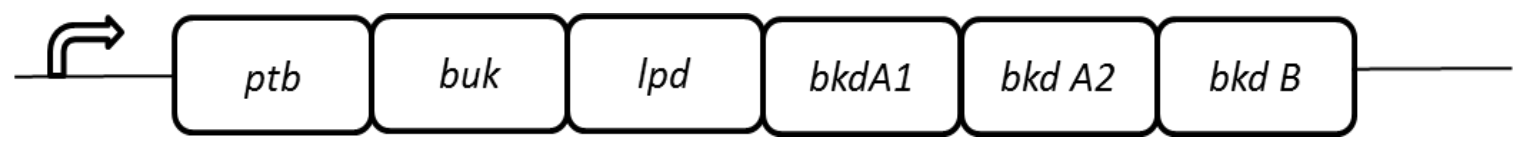

Fig. 1. Structural organization of the $b k d$ operon of L. monocytogenes. Schematic of the $b k d$ operon showing $p t b$, phosphotransbutyrylase; $b u k$, butyrate kinase; $l p d$, dihydrolipoamide dehydrogenase, $b k d A 1$-Bkd E1 $\alpha$ subunit; $b k d A 2$, Bkd E1 $\beta$-subunit; $b k d B$, dihydrolipoamide acetyl transferase [7, 15]. 


\section{Step 1.}

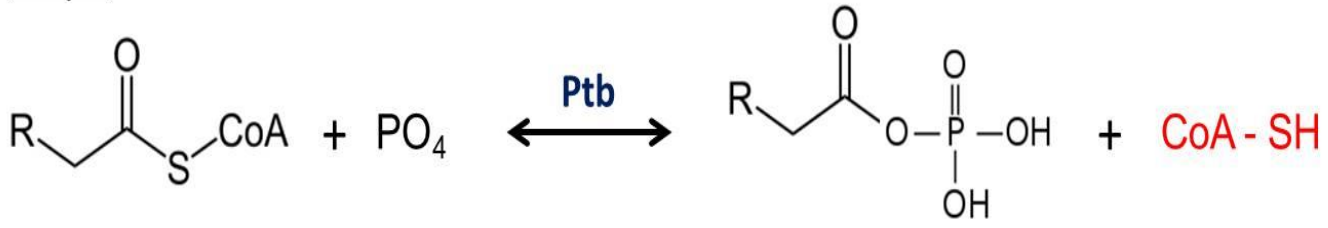

\section{Step 2.}

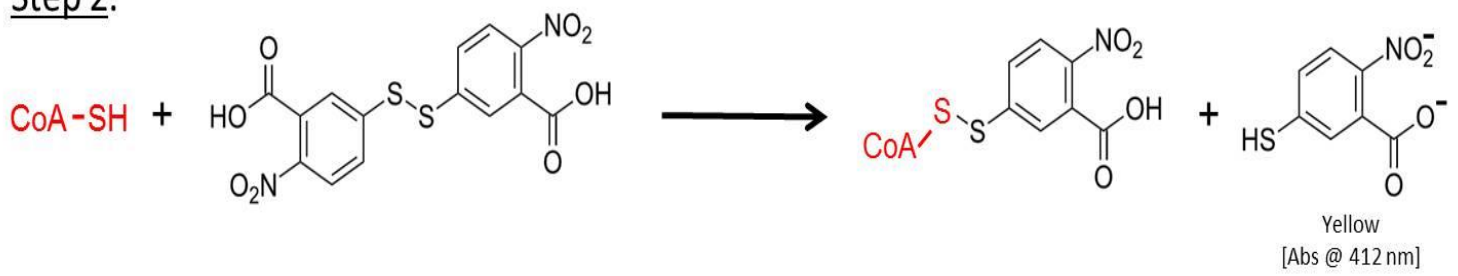

Fig. 2. Reaction catalyzed by Ptb. Step 1 shows the reversible conversion of Acyl-CoA to the corresponding Acyl-phosphate by Ptb activity. Step 2 shows the Ellman reaction of the free thiol on CoA-SH reacting with DTNB (Ellman's reagent) to produce the yellow colored TNB which is used to quantify the reaction. 


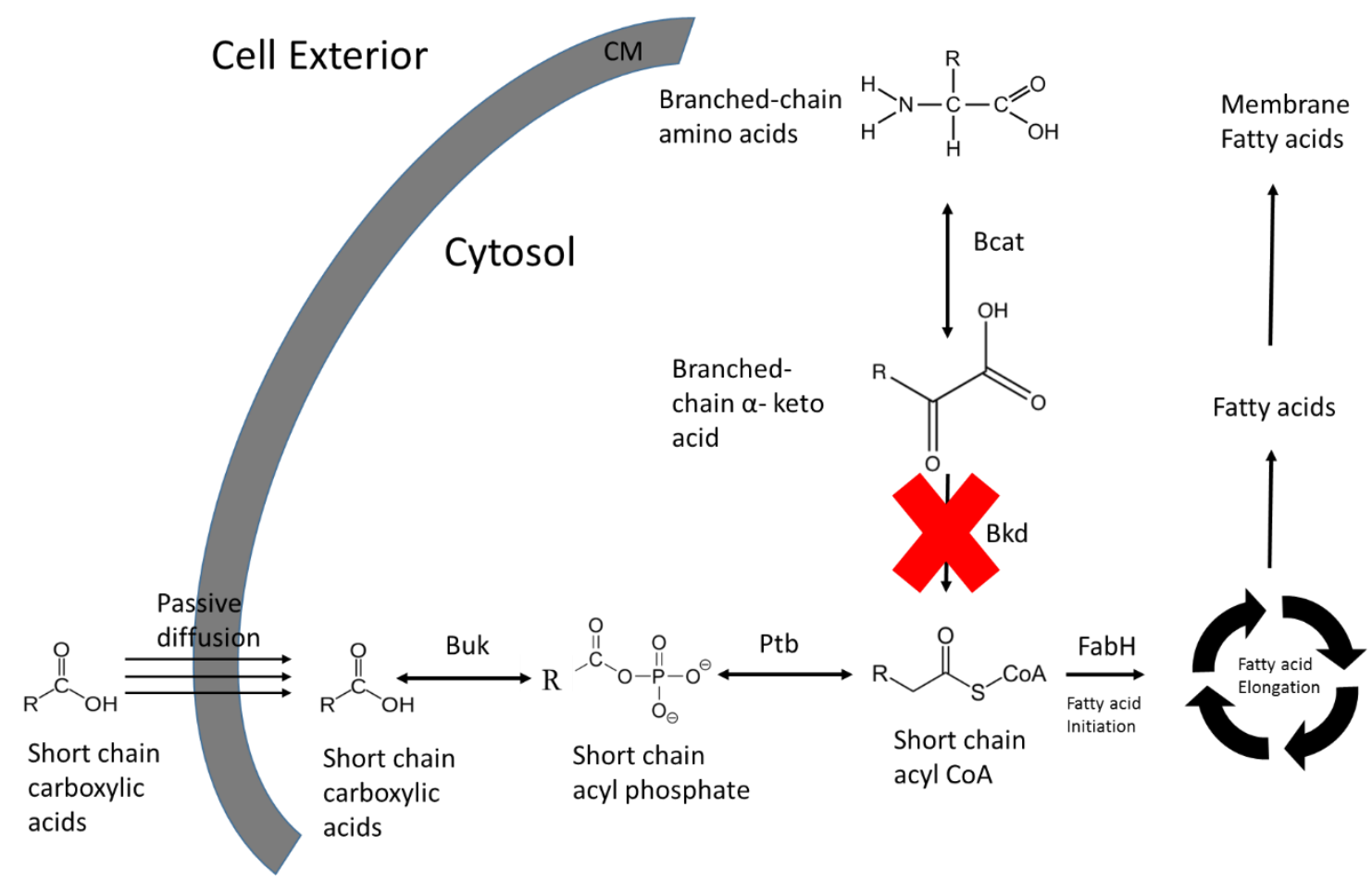

Fig. 3. Proposed alternative pathway for provision of acyl CoA precursors for fatty acid biosynthesis. Schematic showing the sequential conversion of exogenous carboxylic acids into acyl phosphate by the activity of Buk followed by the conversion into acyl CoA by the activity of Ptb and subsequent incorporation of the activated substrates into the membrane fatty acids. 
Step 1

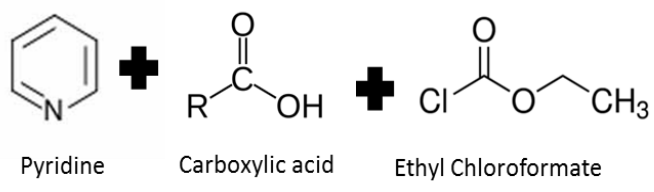

$0.1 \mathrm{~mol}$ of each mixed with ice cold ethyl ether

Step 2

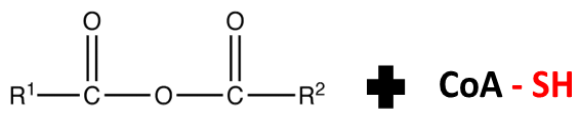

Mixed anhydride

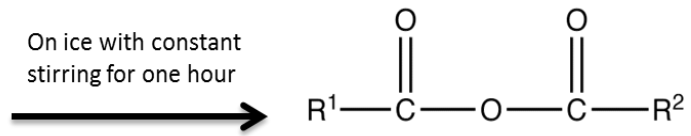

Mixed anhydride

$$
\begin{aligned}
& \text { On ice with } \\
& \text { constant stirring }
\end{aligned}
$$<smiles>[R]CC(=O)SC(=O)OCc1ccccc1</smiles>

Acyl CoA

Fig. 4. Preparation of acyl CoA substrates. Step 1 shows the synthesis of mixed acyl anhydrides from the different carboxylic acids (R group- 2-methyl butyrate and 2-ethyl butyrate). Step 2 shows the reaction of the mixed anhydride with an aqueous solution of CoA resulting in the formation of acyl CoA substrates according to the method outlined by Stadtman [23]. 


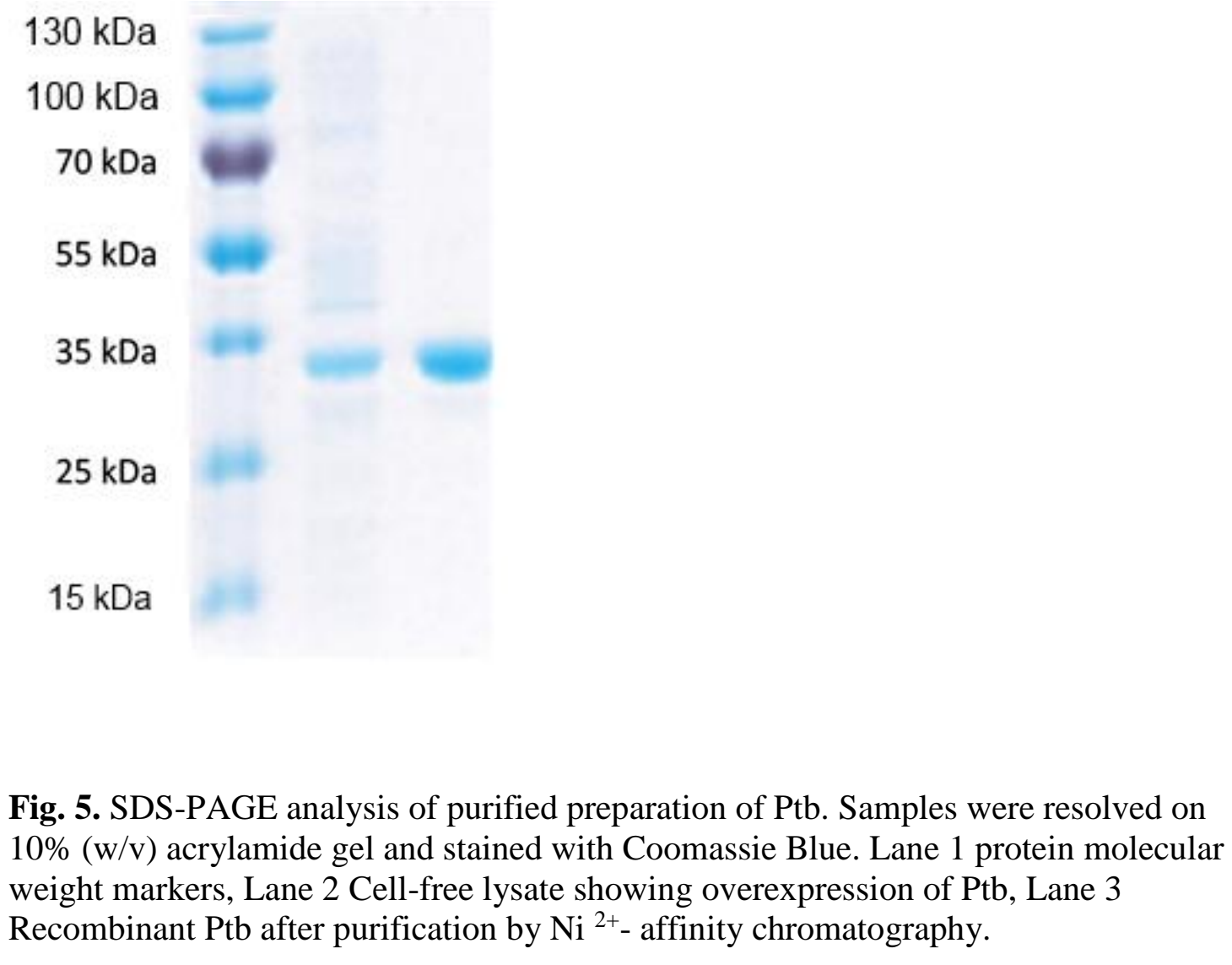




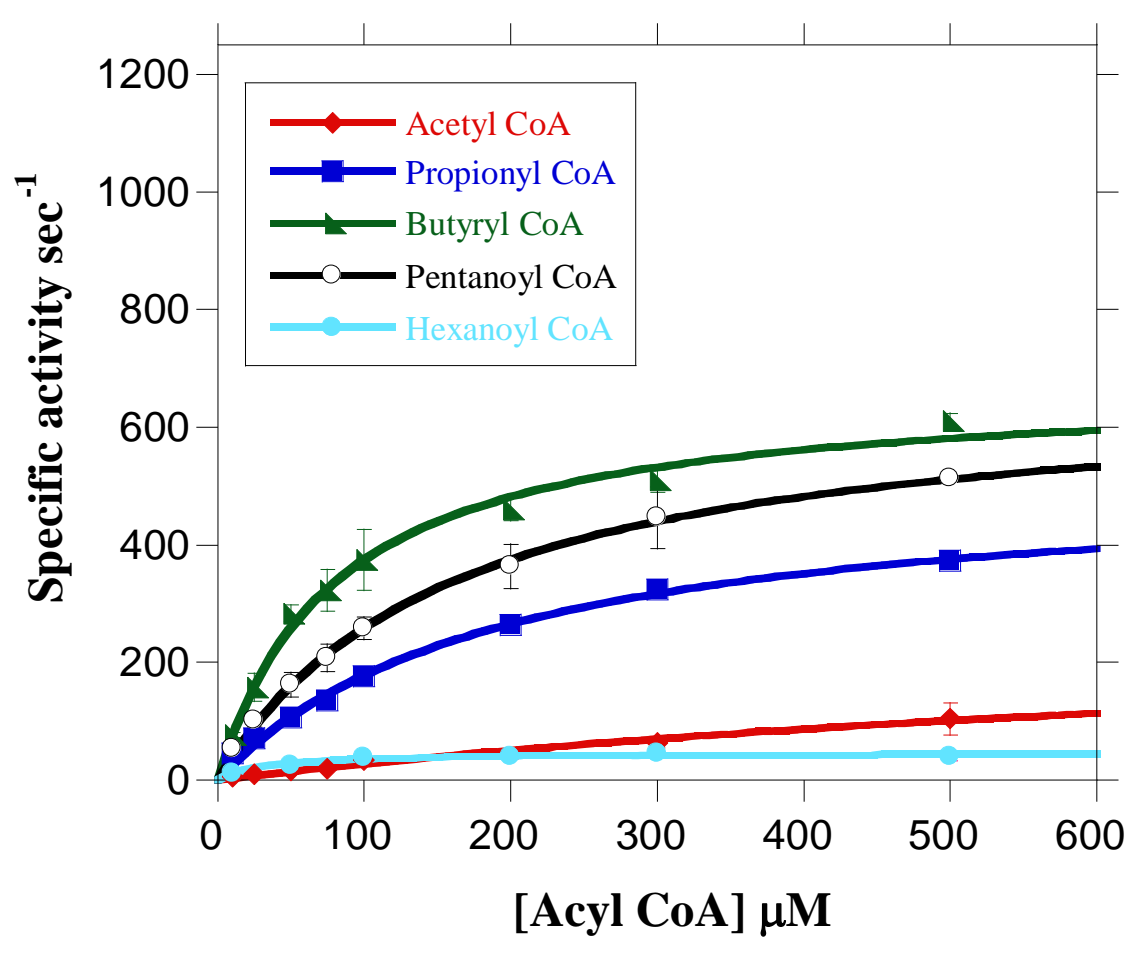

Fig. 6. Concentration-dependence of straight-chain acyl CoA substrates on Ptb activity assayed in the acyl phosphate forming direction. Initial rates for Ptb utilization of the substrates acetyl CoA (closed diamonds), propionyl CoA (open squares), butyryl CoA (closed triangles) and pentanoyl CoA (open circles) and hexanoyl CoA (closed circles) were determined at $25^{\circ} \mathrm{C}$ in the presence of $100 \mathrm{mM}$ inorganic phosphate under standard assay conditions (see Materials and Methods). Data are plotted as a function of substrate concentration and $K_{M}$ and $k_{c a t}$ were determined from fitting the data to the MichaelisMenten equation using Kaleidagraph software. Data are means \pm SEM of at least three experiments. 


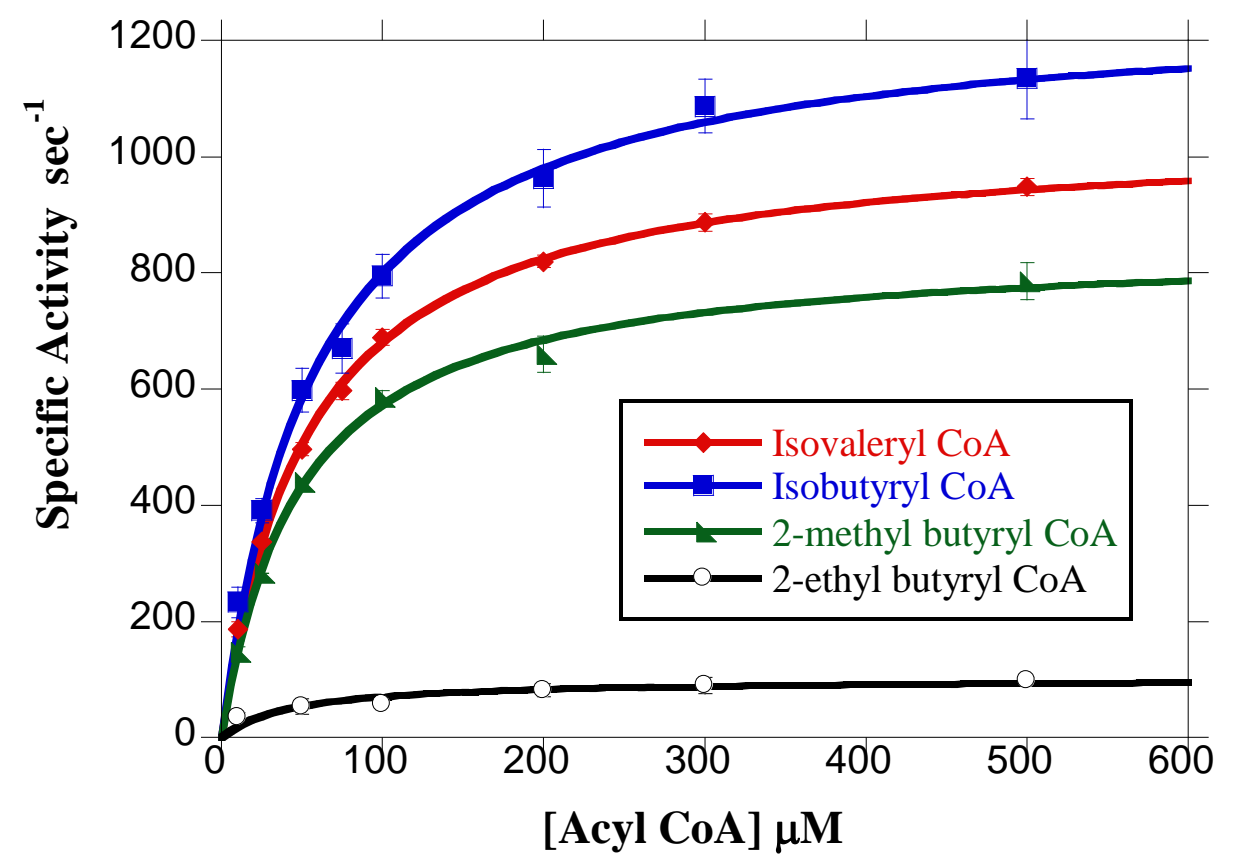

Fig. 7. Concentration-dependence of branched-chain acyl CoA substrates on Ptb activity assayed in the acyl phosphate forming direction. Initial rates for Ptb utilization of the substrates isovaleryl CoA (closed diamonds), isobutyryl CoA (closed squares), 2-methyl butyryl CoA (closed triangles) and 2-ethyl butyryl CoA (open circles) were determined at $25^{\circ} \mathrm{C}$ in the presence of $100 \mathrm{mM}$ inorganic phosphate (see Materials and Methods). Data were plotted as a function of substrate concentration and $K_{M}$ and $k_{c a t}$ were determined from the fit of the data to the Michaelis-Menten equation using Kaleidagraph software. Data plotted are means \pm SEM of at least three experiments. 


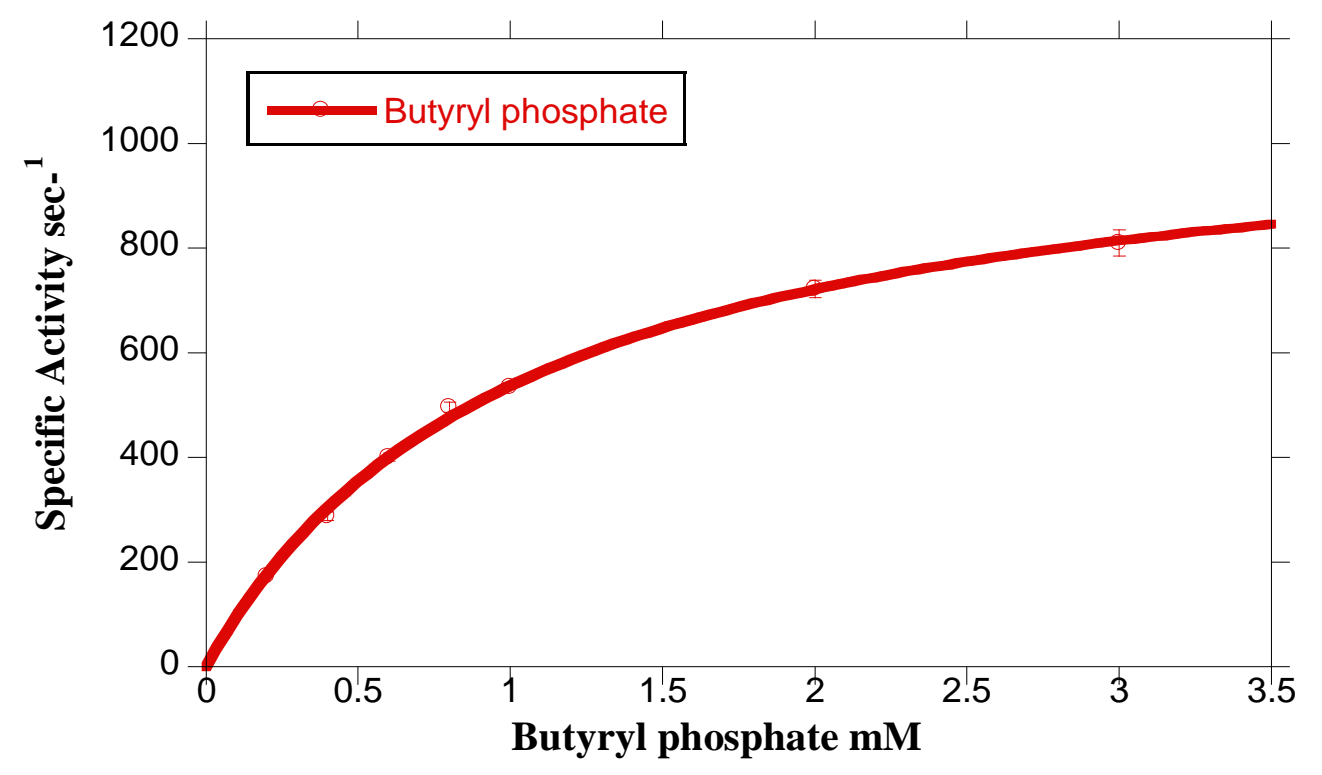

Fig. 8. Concentration-dependence of butyryl phosphate on Ptb activity assayed in the acyl CoA forming direction. Initial rates for Ptb utilization of butyryl phosphate (open circles) were determined at $25^{\circ} \mathrm{C}$ in the presence of $100 \mathrm{mM}$ Tris and $500 \mu \mathrm{M} \mathrm{CoA}$ (see Materials and Methods). Data were plotted as a function of substrate concentration and $K_{M}$ and $k_{c a t}$ were determined from the fit of the data to the Michaelis-Menten equation using Kaleidagraph software. Data plotted are means \pm SEM of three experiments. 


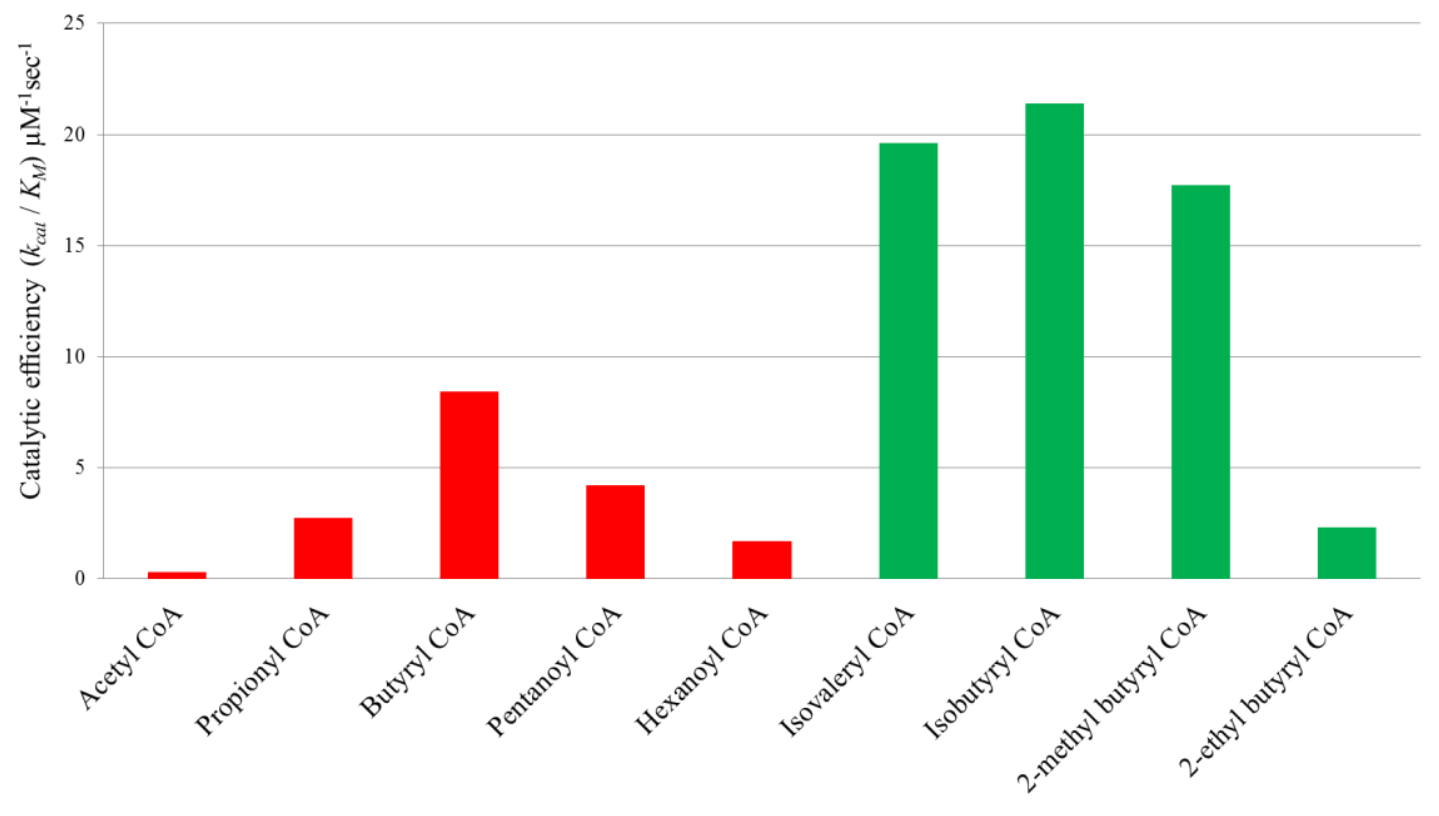

Fig. 9. Comparison of catalytic efficiency of Ptb from L. monocytogenes 10403S with different substrates. Catalytic efficiencies $\left(k_{c a t} / K_{M}\right)$ were determined for the various straight-chain (in red) and branched-chain (in green) carboxylic acid substrates under the standard assay conditions outlined in materials and methods and plotted in a bar graph with increasing chain length and side chain length. 

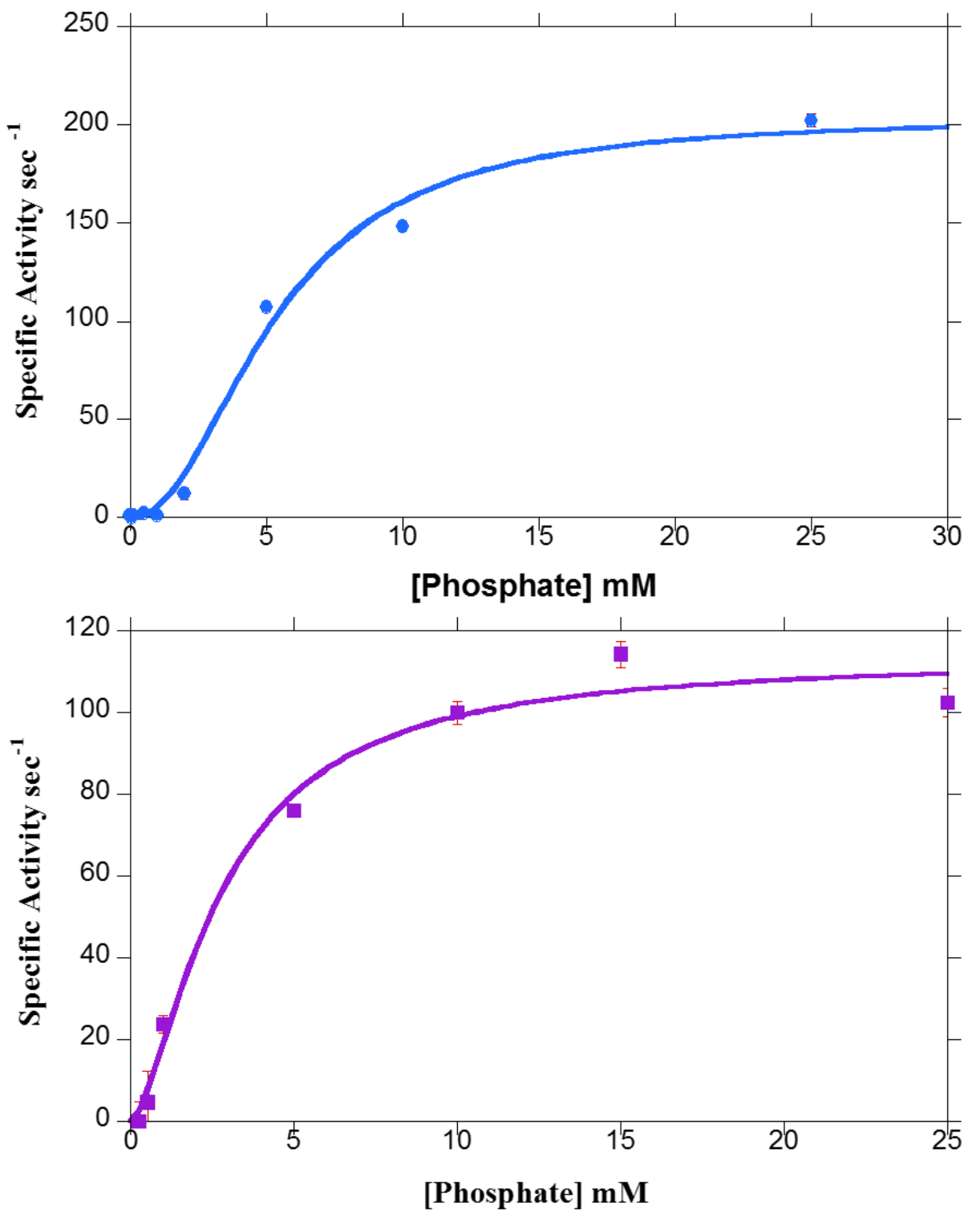

Fig. 10. Concentration dependence of phosphate utilization by $L m$ Ptb. Ptb activity was determined at $25^{\circ} \mathrm{C}$ in the presence of varying phosphate concentrations $(10 \mu \mathrm{M}-25$ $\mathrm{mM})$ and excess A) isobutyryl CoA $(200 \mu \mathrm{M})$ or B $)$ isovaleryl CoA $(200 \mu \mathrm{M})$. Data from three experiments (circles) were fitted to the Hill equation using Kaleidagraph software providing values for $K_{M}$ and $k_{c a t}$. Means of data from at least three experiments (closed squares) \pm SEM are plotted as reaction rate versus phosphate concentration. 


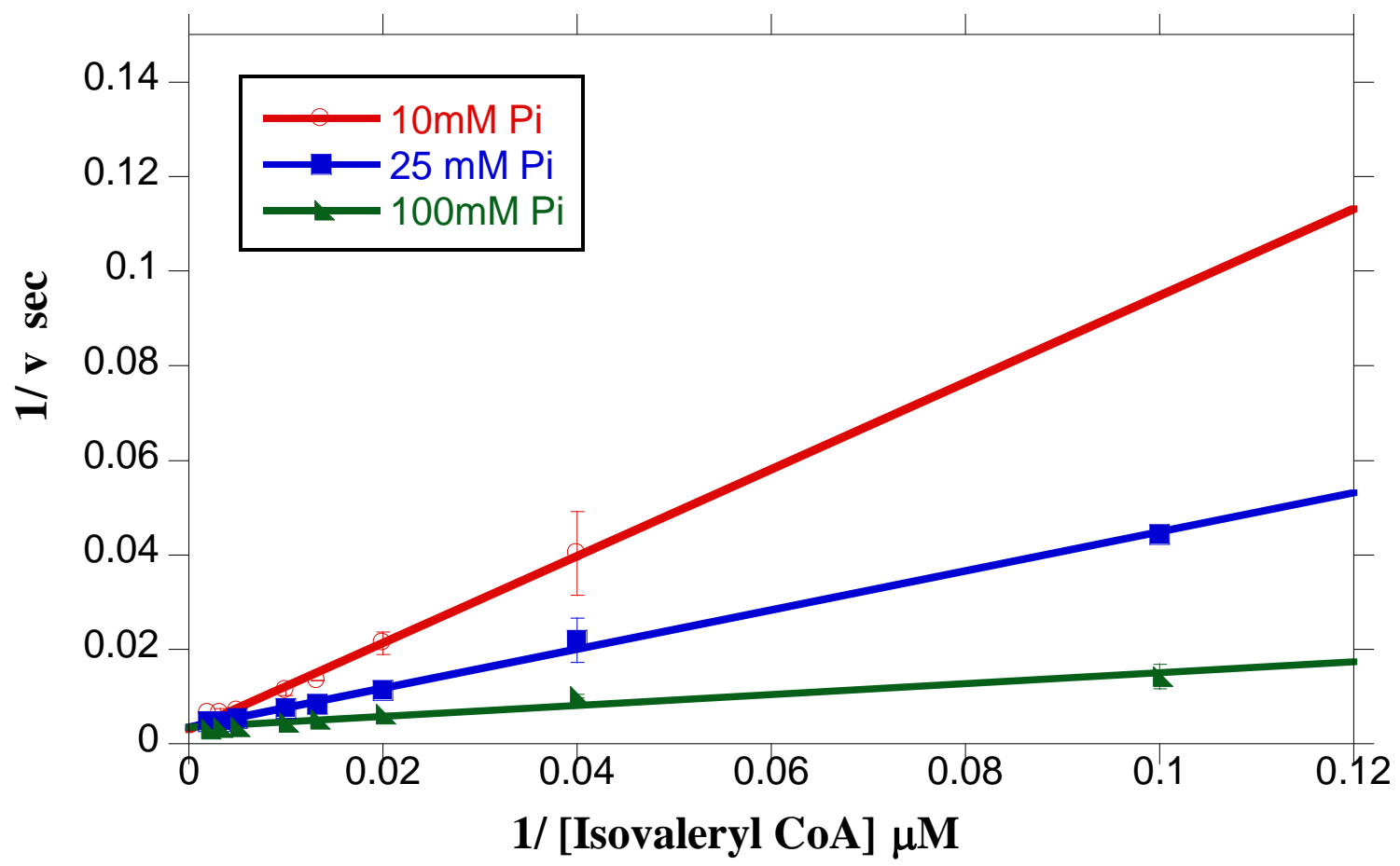

Fig. 11. Ptb activity follows a sequential kinetic model. The concentration dependence of isovaleryl-CoA on Ptb activity was determined in the presence of varying phosphate concentrations, i.e. $10 \mathrm{mM}$ (open circles), $25 \mathrm{mM}$ (closed squares), and $100 \mathrm{mM}$ (closed triangles) under standard assay conditions at $25^{\circ} \mathrm{C}$. Double reciprocal (Lineweaver-Burk) plots of means \pm SEM from at least three experiments at each phosphate concentration were plotted. The three lines clearly intersect, which is indicative of a sequential kinetic mechanism (ping-pong kinetics is characterized by parallel lines). 


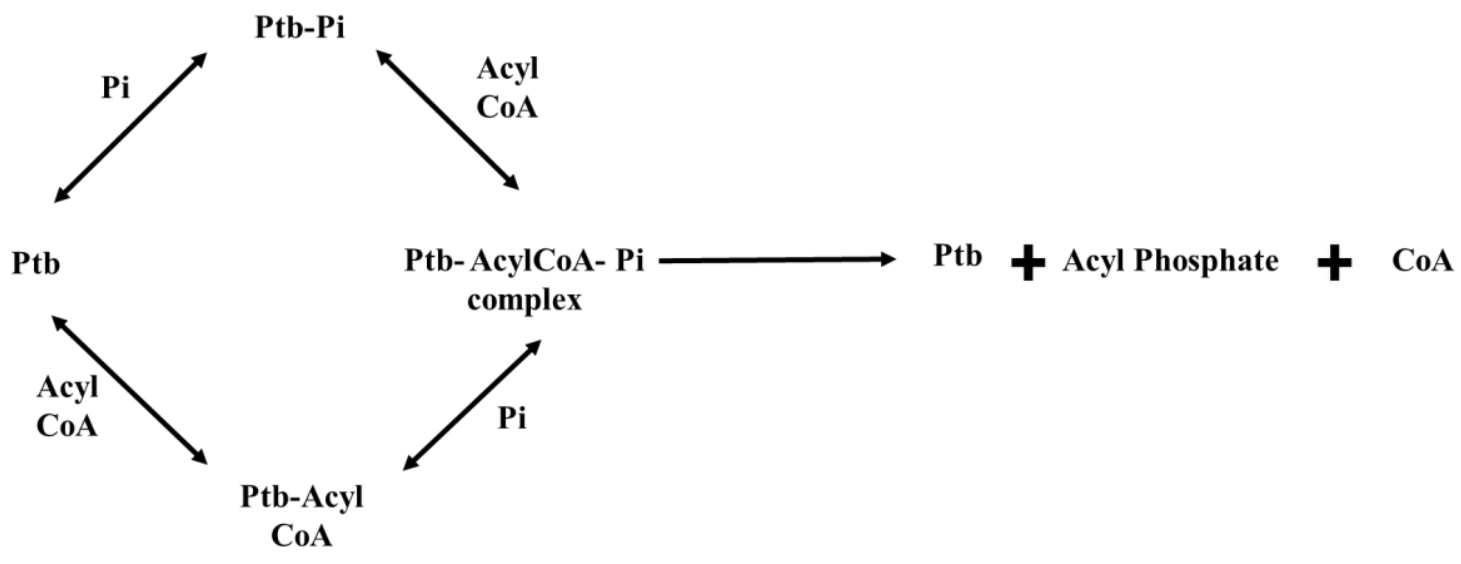

Fig. 12. Mechanism of action of Ptb. Schematic showing the proposed mechanism of action of Ptb involving the sequential binding of the substrates to Ptb to form the ternary complex followed by catalysis and subsequent release of products. 


\section{References}

[1] S.J. Walker, P. Archer, J.G. Banks, Growth of Listeria monocytogenes at refrigeration temperatures, J. Appl. Bacteriol., 68 (1990) 157-162.

[2] M.K. Thomas, R. Vriezen, J.M. Farber, A. Currie, W. Schlech, A. Fazil, Economic cost of a Listeria monocytogenes outbreak in Canada, 2008, Foodborne Pathog. Dis., 12 (2015) fpd.2015.1965.

[3] B.A. Annous, L.A. Becker, D.O. Bayles, D.P. Labeda, B.J. Wilkinson, Critical role of anteiso-C15:0 fatty acid in the growth of Listeria monocytogenes at low temperatures, Appl. Environ. Microbiol., 63 (1997) 3887-3894.

[4] S.K. Mastronicolis, N. Arvanitis, A Karaliota, P. Magiatis, G. Heropoulos, C. Litos, H. Moustaka, a Tsakirakis, E. Paramera, P. Papastavrou, Coordinated regulation of cold-induced changes in fatty acids with cardiolipin and phosphatidylglycerol composition among phospholipid species for the food pathogen Listeria monocytogenes, Appl. Environ. Microbiol., 74 (2008) 4543-4549.

[5] K. Zhu, X. Ding, M. Julotok, B.J. Wilkinson, Exogenous isoleucine and fatty acid shortening ensure the high content of anteiso-C15:0 fatty acid required for lowtemperature growth of Listeria monocytogenes, Appl. Environ. Microbiol., 71 (2005) 8002-8007.

[6] H. Oku, T. Kaneda, Biosynthesis of branched-chain fatty acids in Bacillus subtilis: A decarboxylase is essential for branched-chain fatty acid synthetase, J. Biol. Chem., 263 (1988) 18386-96.

[7] K. Zhu, D.O. Bayles, A. Xiong, R.K. Jayaswal, B.J. Wilkinson, Precursor and temperature modulation of fatty acid composition and growth of Listeria monocytogenes cold-sensitive mutants with transposon-interrupted branched-chain alpha-keto acid dehydrogenase, Microbiology, 151 (2005) 615-23.

[8] Y. Sun, M.X.D. O’Riordan, Branched-chain fatty acids promote Listeria monocytogenes intracellular infection and virulence, Infect. Immun., 78 (2010) 4667-4673.

[9] V.K. Singh, D.S. Hattangady, E.S. Giotis, A.K. Singh, N.R. Chamberlain, M.K. Stuart, B.J. Wilkinson, Insertional inactivation of branched-chain alpha-keto acid dehydrogenase in Staphylococcus aureus leads to decreased branched-chain membrane fatty acid content and increased susceptibility to certain stresses, Appl. Environ. Microbiol., 74 (2008) 5882-90.

[10] M. Julotok, A.K. Singh, C. Gatto, B.J. Wilkinson, Influence of fatty acid precursors, including food preservatives, on the growth and fatty acid composition of Listeria monocytogenes at 37 and 10 degreesC, Appl. Environ. Microbiol., 76 (2010) 142332.

[11] K. Willecke, A.B. Pardee, Fatty acid-requiring mutant of Bacillus subtilis defective in branched chain alpha-keto acid dehydrogenase, J. Biol. Chem., 246 (1971) 526472.

[12] T. Kaneda, Incorporation of Branched-Chain C6 -Fatty acid isomers into the related long-chain fatty acids by growing cells of Bacillus subtilis, Biochemistry, 10 (1970) 340-347. 
[13] S. Sen, S. Sirobhushanam, M.P. Hantak, P. Lawrence, J. Thomas Brenna, C. Gatto, B.J. Wilkinson, Short branched-chain C6 carboxylic acids result in increased growth, novel "unnatural" fatty acids and increased membrane fluidity in a Listeria monocytogenes branched-chain fatty acid-deficient mutant, Biochim. Biophys. Acta - Mol. Cell Biol. Lipids, 1851 (2015) 1406-1415.

[14] M. Debarbouille, R. Gardan, M. Arnaud, G. Rapoport, Role of bkdR, a transcriptional activator of the sigL-dependent isoleucine and valine degradation pathway in Bacillus subtilis, J. Bacteriol., 181 (1999) 2059-2066.

[15] K.E. Nelson, D.E. Fouts, E.F. Mongodin, J. Ravel, R.T. DeBoy, J.F. Kolonay, D.A. Rasko, S. V Angiuoli, S.R. Gill, I.T. Paulsen, J. Peterson, O. White, W.C. Nelson, W. Nierman, M.J. Beanan, L.M. Brinkac, S.C. Daugherty, R.J. Dodson, A.S. Durkin, R. Madupu, et al., Whole genome comparisons of serotype $4 \mathrm{~b}$ and 1/2a strains of the food-borne pathogen Listeria monocytogenes reveal new insights into the core genome components of this species, Nucleic Acids Res., 32 (2004) 2386-2395.

[16] D.E. Ward, R.P. Ross, C.C. van der Weijden, J.L. Snoep, A. Claiborne, Catabolism of branched-chain alpha-keto acids in Enterococcus faecalis: the bkd gene cluster, enzymes, and metabolic route, J. Bacteriol., 181 (1999) 5433-5442.

[17] K.A. Walter, R. V Nair, J.W. Cary, G.N. Bennett, E.T. Papoutsakis, Sequence and arrangement of two genes of the butyrate-synthesis pathway of Clostridium acetobutylicum ATCC 824, Gene, 134 (1993) 107-111.

[18] M.G.N. Hartmanis, Butyrate kinase from Clostridium acetobutylicum, J. Biol. Chem., 262 (1987) 617-621.

[19] C.S. Harwood, E. Canale-Parola, Properties of acetate kinase isozymes and a branched-chain fatty acid kinase from a Spirochete, J. Bacteriol., 152 (1982) 246254.

[20] D.P. Wiesenborn, F.B. Rudolph, E.T. Papoutsakis, Phosphotransbutyrylase from Clostridium acetobutylicum ATCC 824 and its role in acidogenesis, Appl. Environ. Microbiol., 55 (1989) 317-322.

[21] J.W. Cary, D.J. Petersen, E.T. Papoutsakis, G.N. Bennett, G.N. Bennettl, Cloning and expression of Clostridium acetobutylicum phosphotransbutyrylase and butyrate kinase genes in Escherichia coli, J. Bacteriol., 170 (1988) 4613-4618.

[22] M.G.N. Hartmanis, S. Gatenbeck, Intermediary Metabolism in Clostridium acetobutylicum: Levels of enzymes involved in the formation of acetate and butyrate, Appl. Environ. Microbiol., 47 (1984) 1277-1283.

[23] E.R. Stadtman, Preparation and assay of acyl coenzyme A and other thiol esters; use of hydroxylamine, Methods Enzymol., 3 (1957) 931-941.

[24] G. Toennies, J.J. Kolb, Techniques and reagents for paper chromatography, Anal. Chem., 23 (1951) 823-826.

[25] F. Lipmann, L.C. Tuttle, A specific micromethod for the determination of acyl phosphates, J. Biol. Chem., 159 (1945) 21-28.

[26] H.R. Klotzsch, Phosphotransacetylase from Clostridium kluyveri, Methods Enzymol., 13 (1969) 381-386.

[27] G.L. Ellman, Tissue sulfhydryl groups, Arch. Biochem. Biophys., 82 (1959) 70-77. 
[28] J.B. Helms, L.P. Saunders, J. Meyer, C.J. Costa, E. Plowman, N. Williford, M. Corbitt, J.P. Holden, C. Gatto, Kinetic characterization of Na, K-ATPase inhibition by the acetaminophen metabolite, Open J. Mol. Integr. Physiol., 5 (2015) 1-17.

[29] J.R. Brotherus, J. V. Moller, P.L. Jorgensen, Soluble and active renal Na, K-ATPase with maximum protein molecular mass 170,000 + 9,000 daltons; Formation of larger units by secondary aggregation, Biochem. Biophys. Res. Commun., 100 (1981) 146154.

[30] D.K. Thompson, J.S. Chen, Purification and properties of an acetoacetyl coenzyme A-reacting phosphotransbutyrylase from Clostridium beijerinckii ("Clostridium butylicum") NRRL B593, Appl. Environ. Microbiol., 56 (1990) 607-613.

[31] E.-H.H. Aboulnaga, O. Pinkenburg, J. Schiffels, A. El-Refai, W. Buckel, T. Selmer, Effect of an oxygen-tolerant bifurcating butyryl coenzyme A dehydrogenase/electron-transferring flavoprotein complex from Clostridium difficile on butyrate production in Escherichia coli, J. Bacteriol., 195 (2013) 3704-13.

[32] I.H. Segel, Enzyme kinetics: behavior and analysis of rapid equilibrium and steadystate enzyme systems, John Wiley Sons, New York, 1993.

[33] A.K. Singh, Y. Zhang, K. Zhu, C. Subramanian, Z. Li, K. Jayaswal, C. Gatto, C.O. Rock, B.J. Wilkinson, FabH selectivity for anteiso branched-chain fatty acid precursors in low temperature adaptation in Listeria monocytogenes, FEMS Microbiol. Lett., 301 (2009) 1-8.

[34] H.U. Bergmeyer, G. Holz, H. Klotzch, G. Lang, Phosphotransacetylase from Clostridium kluyveri. Culture of the bacterium, isolation, crystallization and properties of the enzyme, Biochem. Z., 338 (1963) 114-21.

[35] A. Bock, J. Glasemacher, R. Schmidt, P. Schönheit, R. Glasemacher, Purification and characterization of two extremely thermostable enzymes, phosphate acetyltransferase and acetate kinase , from the hyperthermophilic eubacterium Thermotoga maritima, J. Bacteriol., 181 (1999) 1861-1867.

[36] T.L. Romick, H.P. Fleming, R.F. McFeeters, Aerobic and anaerobic metabolism of Listeria monocytogenes in defined glucose medium, Appl. Environ. Microbiol., 62 (1996) 304-7.

[37] V.K. Juneja, P.M. Davidson, Influence of altered fatty acid composition on resistance of Listeria monocytogenes to antimicrobials, J. Food Prot., 56 (1993) 302305.

[38] O. Tokarskyy, D.L. Marshall, Mechanism of synergistic inhibition of Listeria monocytogenes growth by lactic acid, monolaurin, and nisin, Appl. Environ. Microbiol., 74 (2008) 7126-9.

[39] C.G.M. Gahan, C. Hill, Listeria monocytogenes: survival and adaptation in the gastrointestinal tract, Front. Cell. Infect. Microbiol., 4 (2014) 9.

[40] J.B. Parsons, T.C. Broussard, J.L. Bose, J.W. Rosch, P. Jackson, C. Subramanian, C.O. Rock, Identification of a two-component fatty acid kinase responsible for host fatty acid incorporation by Staphylococcus aureus, Proc. Natl. Acad. Sci., 111 (2014) 10532-10537. 
[41] J.B. Parsons, M.W. Frank, P. Jackson, C. Subramanian, C.O. Rock, Incorporation of extracellular fatty acids by a fatty acid kinase-dependent pathway in Staphylococcus aureus, Mol. Microbiol., 92 (2014) 234-45.

[42] J.H. Cummings, E.W. Pomare, W.J. Branch, C.P. Naylor, G.T. Macfarlane, Short chain fatty acids in human large intestine, portal, hepatic and venous blood, Gut, 28 (1987) 1221-1227.

[43] Y. Sun, B.J. Wilkinson, T.J. Standiford, H.T. Akinbi, M.X.D. O'Riordan, Fatty acids regulate stress resistance and virulence factor production for Listeria monocytogenes, J. Bacteriol., 194 (2012) 5274-84.

[44] I. Gantois, R. Ducatelle, F. Pasmans, F. Haesebrouck, A. Thompson, J.C. Hinton, F. Van Immerseel, I. Hautefort, Butyrate Specifically Down-Regulates Salmonella Pathogenicity Island 1 Gene Expression, Appl. Environ. Microbiol., 72 (2006) 946949.

[45] T. Tobe, N. Nakanishi, N. Sugimoto, Activation of motility by sensing short-chain fatty acids via two steps in a flagellar gene regulatory cascade in enterohemorrhagic Escherichia coli, Infect. Immun., 79 (2011) 1016-1024.

[46] Y. Zhao, C.A. Tomas, F.B. Rudolph, E.T. Papoutsakis, G.N. Bennett, Intracellular butyryl phosphate and acetyl phosphate concentrations in Clostridium acetobutylicum and their implications for solvent formation, Appl. Environ. Microbiol., 71 (2005) 530-537.

[47] E.L. Milbradt, J.R. Zamae, J.P. Araujo Junior, P. Mazza, C.R. Padovani, V.R. Carvalho, C. Sanfelice, D.M. Rodrigues, A.S. Okamoto, R.L. Andreatti Filho, Control of Salmonella enteritidis in turkeys using organic acids and competitive exclusion product, J. Appl. Microbiol., 117 (2014) 554-563.

[48] C. Fernández-Rubio, C. Ordóñez, J. Abad-Gonzalez, A. Garcia-Gallego, M.P. Honrubia, J.J. Mallo, R. Balaña-Fouce, C. Fernandez-Rubio, C. Ordonez, R. BalanaFouce, Butyric acid-based feed additives help protect broiler chickens from Salmonella enteritidis infection, Poult. Sci., 88 (2009) 943-948.

[49] S.H. Lawrence, J.G. Ferry, Steady-state kinetic analysis of phosphotransacetylase from Methanosarcina thermophila, 188 (2006) 1155-1158.

[50] J. Henkin, R.H. Abeles, Evidence against an acyl-enzyme intermediate in the reaction catalyzed by clostridial phosphotransacetylase, Biochemistry, 15 (1976) $3472-3479$. 
CHAPTER II

UTILIZATION OF MULTIPLE SUBSTRATES BY BUTYRATE KINASE

FROM LISTERIA MONOCYTOGENES

\section{Highlights}

- Buk from L. monocytogenes exhibited broad substrate specificity

- Buk preferred substrates with a chain length of 3 to 5 carbons

- An alkyl side chain improved binding among shorter chain substrates

- Buk could utilize unnatural substrates such as 2-ethylbutyrate, 2-methylpentanoate and 3-methylpentanoate

- Buk catalysis involved ternary complex formation

\section{Keywords}

Butyrate kinase, Branched-chain carboxylic acids, Branched-chain fatty acids, Fatty acid biosynthesis, Membrane fatty acid composition, Acyl phosphate 


\section{ABSTRACT}

Listeria monocytogenes, the causative agent of listeriosis, can build up to dangerous levels in refrigerated foods potentially leading to expensive product recalls. An important aspect of the bacterium's growth at low temperatures is its ability to increase the branched-chain fatty acid anteiso C15:0 content of its membrane at lower growth temperatures, which imparts greater membrane fluidity. Mutants in the branched-chain $\alpha$ keto dehydrogenase $(b k d)$ complex are deficient in branched-chain fatty acids (BCFAs,) but these can be restored by feeding $\mathrm{C} 4$ and $\mathrm{C} 5$ branched-chain carboxylic acids (BCCAs). This suggests the presence of an alternate pathway for production of acyl CoA precursors for fatty acid biosynthesis. We hypothesize that the alternate pathway is composed of butyrate kinase (buk) and phosphotransbutyrylase ( $p t b)$ encoded in the $b k d$ complex which produce acyl CoA products by their sequential action through the metabolism of carboxylic acids. We determined the steady state kinetics of recombinant His-tagged Buk using 11 different straight-chain and BCCA substrates in the acyl phosphate forming direction. Buk demonstrated highest catalytic efficiency with pentanoate as the substrate. Low product formation observed with acetate $(\mathrm{C} 2)$ and hexanoate (C6) as the substrates indicates that Buk is not involved in either acetate metabolism or long chain carboxylic acid activation. We were also able to show that Buk catalysis occurs through a ternary complex intermediate. Additionally, Buk demonstrates a strong preference for BCCAs at low temperatures. These results indicate that Buk maybe involved in the activation and assimilation of exogenous carboxylic acids for membrane fatty acid biosynthesis. 


\section{Introduction}

Listeria monocytogenes is the dangerous foodborne pathogen that causes listeriosis, a disease characterized by gastroenteritis, meningitis, spontaneous miscarriages and high mortality rate among infected individuals [1]. L. monocytogenes grows actively at temperatures as low as $-0.1{ }^{\circ} \mathrm{C}$, building up to dangerous levels, which is enabled by its highly fluid membrane [2]. The cellular membrane of L. monocytogenes is enriched with branched-chain fatty acids (BCFAs) which have greater cross sectional area, disrupt the close packing of membrane fatty acids and thus increase the fluidity of the membrane $[3,4]$. L. monocytogenes further increases the membrane content of anteiso C15:0, a survival strategy which supports its growth at low temperatures $[2,5,6]$.

Expensive food product recalls due to outbreaks of Listeria underscore the growing need for better control strategies for this organism (http://www.cdc.gov/listeria/outbreaks).

BCFAs, which make up $>90 \%$ of membrane fatty acid content of $L$.

monocytogenes, are biosynthesized by the activity of branched-chain amino transferase (Bcat) and branched-chain $\alpha$-keto acid dehydrogenase (Bkd) on branched chain amino acids (BCAAs), followed by elongation of the catabolic products by the bacterial fatty acid biosynthesis pathway (FAS II) [7]. Disruption of this pathway, as in the case of mutants of L. monocytogenes lacking functional Bkd (cld-1, cld-2/ MOR401), results in reduced fitness and impairment of the homeoviscous adaptation strategies employed by the organism when exposed to low growth temperatures $[4,5,8,9]$. Such mutants are deficient in membrane BCFA content, unable to grow at low temperatures, and are significantly affected by unfavorable environmental conditions $[4,5,8,10]$. Addition of 2- 
methyl butyrate, the precursor of anteiso C15:0, restores the membrane BCFA content and thus rescues the growth of the mutant and leads to greater survival in macrophages and at low temperatures, as well as greater tolerance for acidity and alkalinity [4,9-12]. Restoration of membrane BCFA content by the addition of 2-methylbutyrate has also been observed in bkd mutants of Staphylococcus aureus and Bacillus subtilis [13,14].

Comprehensive investigation of media supplementation conducted by Kaneda [15] in B. subtilis and Sen et al. [16] in L. monocytogenes show that a wide range of carboxylic acids, which include straight chain carboxylic acids (SCCAs) such as propionate and butyrate and branched-chain carboxylic acids (BCCAs, including 2methylbutyrate and isobutyrate) are capable of alteration of membrane fatty acid composition. Additionally, supplementation of unnatural BCCAs such as 2-ethylbutyrate and 2-methylpentanoate result in the incorporation of novel fatty acids in the membrane arising from these compounds $[11,15,16]$. Since the only known source of BCFAs is via biosynthesis, it is presumed that these compounds are converted into their thioester derivatives by a pathway hitherto uncharacterized, and are utilized as primers by $\beta$-keto acyl ACP synthase III (FabH), the enzyme catalyzing the first condensation step in FAS II pathway [17]. The alternate pathway, first suggested by Willecke and Pardee in $B$. subtilis [14], must therefore exhibit broad substrate specificity to fulfill the requirements of activation of such a large range of substrates.

Homology of the L. monocytogenes bkd operon to the bkd operon in Enterococcus faecalis led to the identification of two genes butyrate kinase (buk) and phosphotransbutyrylase ( $p t b)$ present upstream of the lpd gene in the $b k d$ operon [18-20]. 
Ptb is known to catalyze the reversible conversion of acyl CoA compounds into their corresponding acyl phosphate derivatives [21]. Ptb from L. monocytogenes demonstrates broad substrate specificity and shows a strong preference for branched-chain substrates (Sirobhushanam et al., 2016). Buk catalyzes the phosphorylation of a variety of carboxylic acids and is a BCCA kinase in Spirochaeta isovalerica MA-2 [22,23]. Although the Ptb-Buk pathway has been shown to be the source of butyrate secretion in the important industrial organism Clostridium acetobutylicum, we hypothesize that presence of significant concentrations of exogenous carboxylic acids induces the formation of the acyl CoA products by the sequential activity of Buk and Ptb which are subsequently elongated by the FAS II pathway.

Buk is well characterized in the industrial fermentor, C. acetobutylicum, in which it is an important source of ATP during the acidogenesis stage of fermentation [23]. Other roles such as catabolism of branched-chain amino acids and ATP generation for survival have been attributed to Buk in E. faecalis and S. isovalerica [22,24]. We determined the substrate specificities of Buk by in vitro assay of the recombinant enzyme to investigate the potential role of Buk from L. monocytogenes in the phosphorylation of exogenous carboxylic acids for provision of precursors of fatty acid biosynthesis. Our results indicate that Buk is capable of conversion of a large number of carboxylic acids into their acyl phosphate derivatives. 


\section{Materials and methods}

\subsection{Materials}

All the materials including antibiotics used as selection agents and acetate kinase were purchased from Sigma-Aldrich (St. Louis, MO).

\subsection{Cloning and expression of Buk}

Genomic DNA of L. monocytogenes 10403S, grown in Brain Heart Infusion (BHI) media (Becton Dickinson, Sparks, MD), was isolated using a Masterpure genomic DNA purification kit according to the manufacturer's instructions (Epicenter, Madison, WI). The $b u k$ gene was amplified using a $b u k$ forward primer with a SacI restriction site (bukF 5'ATGCGAGCTCATGTCTTTTGATGTTTT) and reverse primer with a PstI restriction site (bukR 5' ATGCCTGCAGTTAGTACTCTTTTTCTT), which were designed based on the sequence of L. monocytogenes strain EGDe. The restriction sites were used for ligation of the buk gene into the expression vector pRSETa (Thermofisher, Waltham, MA) using T4 DNA ligase (Fermentas, Waltham, MA). Transformation of the plasmid pRSETa-buk into competent $E$. coli BL21 (DE3) cells was followed by confirmation of overexpression of the protein by Western blotting. DNA purification, ligation and transformation were performed according to the manufacturer's instructions (Qiagen, Valencia, CA). Ampicillin $(50 \mu \mathrm{g} / \mathrm{ml})$ was used as the selection agent for growth of pRSETa and pRSETa-buk carrying E. coli cells in Luria broth (Becton Dickinson, Sparks, MD). Overnight culture of cells carrying the pRSETa-buk vector was diluted into $500 \mathrm{ml}$ of Luria broth until the $\mathrm{OD}_{600}$ reached 0.6. Addition of isopropyl $\beta$-Dthiogalactopyranoside (IPTG) to a final concentration of $3 \mathrm{mM}$ was used to induce the 
overexpression of Buk and the culture was incubated at $37^{\circ} \mathrm{C}$ with shaking at $200 \mathrm{rpm}$ for 3 hours. Cell pellets were obtained by centrifugation at $4{ }^{\circ} \mathrm{C}$ at $3,000 \mathrm{~g}$ and were stored at $-80{ }^{\circ} \mathrm{C}$ until use.

\subsection{Purification of Buk}

The cell pellet was resuspended in $25 \mathrm{ml}$ binding buffer $(200 \mathrm{mM} \mathrm{NaCl}, 25 \mathrm{mM}$ $\mathrm{N}$-(2-Hydroxyethyl) piperazine-N'-(2-ethanesulfonic acid) [HEPES], $1 \mathrm{mM} \mathrm{MgCl}_{2}, 5$ $\mathrm{mM}$ imidazole, $5 \%$ glycerol and $5 \mathrm{mM} \beta$-mercaptoethanol $\mathrm{pH} 7.5)$. The cells were broken using a French press at 16,000 psi and two passes through the machine. Cell debris was removed by centrifugation at 20,000 $\mathrm{g}$ for $30 \mathrm{~min}$ and the cell-free extract was allowed to bind by gravity flow with nickel-chelated nitrilotriacetic acid $\left(\mathrm{Ni}^{2+}-\mathrm{NTA}\right)$ resin (Thermo Scientific, Waltham, MA), which was pretreated with binding buffer. The resin was then washed with 5 column volumes of wash buffer (200 mM NaCl, $25 \mathrm{mM}$ HEPES, $1 \mathrm{mM}$ $\mathrm{MgCl}_{2}, 10 \mathrm{mM}$ imidazole, 5\% glycerol and $5 \mathrm{mM} \beta$-mercaptoethanol, $\mathrm{pH}$ 7.5). The bound His6-tagged Buk was then eluted with $4 \mathrm{ml}$ of elution buffer $(200 \mathrm{mM} \mathrm{NaCl}, 25$ $\mathrm{mM}$ HEPES, $1 \mathrm{mM} \mathrm{MgCl}$, $250 \mathrm{mM}$ imidazole, $25 \%$ glycerol and $5 \mathrm{mM} \beta$ mercaptoethanol, $\mathrm{pH}$ 7.5). The concentration of protein was determined by the Bradford assay using bovine serum albumin as the standard (Biorad, Hercules, CA). Addition of $\beta$ mercaptoethanol (4 mM) and glycerol (25\%) was essential for the catalytic activity of Buk. Analysis of the preparation of Buk by sodium dodecyl sulfate-polyacrylamide gel electrophoresis (SDS-PAGE) confirmed the calculated molecular mass of Buk $(\sim 42$ $\mathrm{kDa}$ ) and the purity of the enzyme preparation (Fig. 1). 


\subsection{Standardization of Buk assay}

Buk activity was measured in the acyl phosphate forming direction based on the assay developed by Lipmann and Tuttle [25] as described by Hartmanis [23] with minor modifications. Buk catalyzes the reversible transfer of the $\gamma$-phosphate of ATP to the carbonyl group of the carboxylic acid resulting in the formation of the high energy compound acyl phosphate as the product (Fig.2. step 1). The acyl phosphate product was quantitated by the inclusion of neutralized hydroxylamine in the reaction mixture thereby resulting in the formation of its corresponding stable acyl hydroxamate (Fig. 2. Step 2). Acyl hydroxamate in the presence of acidic ferric chloride forms a purple ferric hydroxamic acid complex which was quantified spectrophotometrically at $540 \mathrm{~nm}$. Lipmann and Tuttle [25] analyzed a variety of compounds and showed that color formation was specific for acyl anhydride compounds and color intensity did not vary with differences in the chain length of the compounds.

Commercial acetate kinase was utilized to standardize assay conditions. All assays of Buk activity were performed at $37{ }^{\circ} \mathrm{C}$ in a final volume of $200 \mu \mathrm{l}$. Bukdependent formation of the corresponding acyl phosphate product was then studied in the presence of varying concentrations of carboxylic acid substrates and ATP. The assay medium contained $50 \mathrm{mM}$ Tris-hydrochloride buffer ( $\mathrm{pH} 7.5$ ), $4 \mathrm{mM} \mathrm{MnSO}$, $10 \mathrm{mM}$ ATP, $400 \mathrm{mM}$ neutralized hydroxylamine, $5 \mathrm{mM} \beta$-mercaptoethanol and varying amounts of carboxylic acid $(100 \mu \mathrm{M}$ to $300 \mathrm{mM})$. The reaction was initiated with the addition of purified Buk (1-5 $\mu \mathrm{M}$ final concentration) and arrested by the addition of 40 $\mu 1$ of $50 \%$ trichloroacetic acid (TCA). The precipitated protein was removed by 
centrifugation at $13,000 \mathrm{~g}$ for 2 minutes and the supernatant was transferred to a fresh tube. Color was developed by the addition of $400 \mu \mathrm{l}$ of $1.25 \%$ ferric chloride solution in 1M hydrochloric acid and the intensity was measured using a Beckman DU-65 spectrophotometer at $540 \mathrm{~nm}$. Initial reaction rates were calculated as the amount of product formed per minute. Acetyl phosphate at varying concentrations $(250 \mu \mathrm{M}$ to 5 $\mathrm{mM}$ ) was used to determine a standard curve. Assay medium without Buk was utilized as the blank.

\subsection{Kinetic analysis of Buk}

Initial velocities of Buk in the presence of varying concentrations of the carboxylic acid substrates were determined and plotted as a function of the substrate concentration. Non-linear curve fitting of the data to the Michaelis-Menten equation, $v=$ $k_{\text {cat }} *[\mathrm{~S}] /\left(K_{0.5}+[\mathrm{S}]\right)$ where $k_{\text {cat }}$ is the turnover number and $K_{0.5}$ is the substrate concentration that produces half maximal velocity and $[\mathrm{S}]$ is the substrate concentration. However, inhibition of product formation was observed at higher concentrations of substrate in the case of hexanoate, 2-ethyl butyrate, 2-methylpentanoate and 3methylpentanoate. Steady state kinetic parameters were computed for these substrates using KaleidaGraph software by fitting the data to the Michaelis-Menten equation incorporating substrate inhibition, $v=k_{c a t} *[\mathrm{~S}] /\left(K_{0.5}+[\mathrm{S}] *\left(1+[\mathrm{S}] /\left[\mathrm{S}_{\mathrm{i}}\right]\right)\right)$ where all variables are the same with the additional factor $S_{i}$ which is the substrate concentration that produces inhibition to half maximal velocity (Kaleidagraph, Synergy software, Reading, PA). Fits to this equation were used to determine the kinetic constants $\left(K_{0.5}\right.$ and $\left.k_{\text {cat }}\right)$. At least three replicates of each assay were conducted and the results were 
presented as means \pm SEM. The apparent $K_{0.5}$ and $k_{c a t}$ values were determined for a variety of straight-chain and branched-chain carboxylic acid compounds listed in Table 1.

\section{Results and discussion}

Butyrate kinase (EC 2.7.2.7) belongs to the ASKHA (acetate and sugar kinases / heat shock cognate / actin) superfamily of phosphotransferases and catalyzes the reversible phosphorylation of short- chain carboxylic acids in C. acetobutylicum [23]. The focus of this study was to investigate the substrate preferences of Buk from $L$. monocytogenes to determine if it was capable of phosphorylating the range of SCCAs and BCCAs which have induced alteration of the membrane fatty acid composition. To this end, Buk heterologously expressed in E. coli was purified by its N-terminal His-tag by affinity purification and assayed for activity by the hydroxamate method. Kinetic constants of Buk were determined in the presence of saturating concentration of ATP (10 $\mathrm{mM})$ and varying concentrations $(0.1-300 \mathrm{mM})$ of various SCCAs and BCCAs.

\subsection{Buk demonstrates broad substrate specificity}

\subsubsection{SCCAs}

In order to understand the structural limits of substrates preferred by $L m B u k$, its activity was analyzed with several SCCAs with differing chain lengths (C2-C6). Among

the SCCAs that were tested, Buk showed the highest catalytic efficiency $\left(5.5 \mathrm{mM}^{-1} \mathrm{~min}^{-1}\right)$ and affinity for pentanoate (C5) with an apparent $K_{0.5}$ of $13.1 \mathrm{mM}$. Butyrate (C4) also served as a good substrate with a corresponding $K_{0.5}$ value of $50.5 \mathrm{mM}$ (Fig. 3A \& Table 1). Buk activity with propionate (C3) showed that substantial product formation was also associated with lower affinity $\left(K_{0.5}=174.1 \mathrm{mM}\right)$ compared to $\mathrm{C} 4$ and C5 SCCAs. The 
stronger preference for pentanoate demonstrated by $L m$ Buk was similar to the branchedchain fatty acid kinase from $S$. isovalerica [22]. Additionally, the $K_{0.5}$ values exhibited by $L m B u k$, were relatively higher than reported values for $S i B u k$ utilizing butyrate and propionate (Fig. 3A \& Table 1) [22,23].

Acetate (C2) and hexanoate (C6) were poor LmBuk substrates (Fig. 3A \& Table 1). However, elevated concentrations of acetate $(>80 \mathrm{mM})$ did result in measurable product formation ( $10 \%$ of the product with propionate as the substrate) (data not shown). Extremely low activity with acetate indicates that Buk is not involved in the secretion of acetate observed during aerobic and anaerobic growth in L. monocytogenes [26]. Furthermore, Buk is unlikely to be involved in phosphorylation of long chain fatty acids judging by the similarly low activity with hexanoate (C6) as the substrate. Thus, the modest integration of medium and long chain fatty acids reported earlier probably occurs via an alternate pathway [27].

These data indicate the broad substrate specificity and chain length preference (C3-C5) of LmBuk. The substantial activity in the phosphorylation direction (Fig. 3A and 3B; Table 1) supports our hypothesis that presence of sufficient concentrations of carboxylic acid precursors could drive the reaction in the acyl phosphate forming direction. Furthermore, L. monocytogenes Ptb has been shown to form butyryl-CoA from butyryl phosphate thus emphasizing the viability of the reversibility of this pathway [28]. The reversibility of the Ptb-Buk pathway has been utilized in vitro for the production of polyhydroxy alkanoic acids in C. acetobutylicum and Bacillus megaterium and the 
authors demonstrate the importance of broad substrate specificity of the Ptb-Buk pathway in this context $[29,30]$.

It is also likely that the phosphorylated products of carboxylic acids serve a purpose other than formation of fatty acids. Butyrate is well known as an effector of gene expression. For example, butyrate causes an upregulation of virulence factor expression in enterohemorrhagic E. coli (EHEC) [31]. On the other hand, downregulation of virulence factor expression caused by exposure to butyrate has been observed in Salmonella enterica Enteritidis and L. monocytogenes [10,32]. Intracellular peak concentrations of butyryl phosphate, which likely behaves as a small molecule phosphate donor, are accompanied by altered expression of a large set of genes in C. acetobutylicum $[33,34]$. Thus, it is probable that Buk plays a significant role in signal transduction in $L$. monocytogenes, since the organism encounters high concentrations of short chain fatty acids in the mammalian gut during infection [35].

\subsubsection{BCCAs}

Buk from the marine spirochete MA-2 has been reported to be a true branchedchain fatty acid kinase [22]. We sought to determine if $L m$ Buk exhibited similar behavior and kinetic constants were determined with various BCCAs differing in the size and branch position to this end. All BCCAs tested proved to be good substrates for LmBuk. Curiously, the presence of a methyl branch increased affinity of substrates which had a shorter chain length. For example, the $K_{0.5}$ values for propionate $(174.1 \mathrm{mM})$ was $\sim 20$ fold higher than that of isobutyrate (C3 with a methyl branch at the $2^{\text {nd }}$ position) (7.6 $\mathrm{mM}$ ) revealing a substantial increase in affinity associated with the branched chain. A 
modest improvement in affinity was observed with the $\mathrm{C} 4$ substrates as demonstrated by a comparison of the $K_{0.5}$ for butyrate with that for isovalerate, 2-methylbutyrate and 2ethylbutyrate (Fig. 3B \& Table 1). However, the data derived from branched C5 substrates (2-methyl pentanoate and 3-methyl pentanoate) support lower binding affinity when compared with their straight chain counterparts (Fig. 3B \& Table 1). Although the presence of the branch appears to increase affinity it also seems to cause a concomitant reduction in the turnover rate (Fig. 3B \& Table $1 \& 2$ ). Such a change might indicate that branched chain substrates have a reduced off-rate which would produce both of these observed enzymatic characteristics.

Although Buk activity is likely important in the incorporation of these compounds into the L. monocytogenes fatty acid pool, factors such as the substrate specificity of Ptb, $\mathrm{FabH}$, and the other enzymes that participate in phospholipid biosynthesis probably play a larger role. Consistent with this is that although Buk showed the best catalytic efficiency with pentanoate, Ptb from L. monocytogenes prefers BCCA substrates, and does not utilize pentanoyl-CoA efficiently [28,36]. Additionally, synthesis of BCFAs has been reported to be dependent on the substrate specificity of $\mathrm{FabH}$, which catalyzes the first committed step in fatty acid biosynthesis, thus these endogenous substrates likely outcompete the products of the Buk-Ptb pathway [37].

\subsubsection{ATP}

The ATP concentration dependence $(10 \mu \mathrm{M}-15 \mathrm{mM})$ for $L m B u k$ was determined in the presence of $200 \mathrm{mM}$ butyrate by fitting the data to the Michaelis-Menten equation as described in the "Materials and Methods". The $K_{0.5 A T P}$ for $L m B u k$ was $3.18 \mathrm{mM}$ (Fig. 
4), values which are in agreement with Buk from C. acetobutylicum and the marine spirochete $S$. isovalerica [22,23]. Additionally, presence of $2 \mathrm{mM}$ ADP demonstrated competitive inhibition of the binding of ATP with an increase in the $K_{0.5 A T P}$ to $6.09 \mathrm{mM}$ (Fig. 4).

\subsection{Buk utilized unnatural BCCAs as its substrates}

LmBuk also exhibited significant product formation with unnatural BCCAs such as 2-ethyl butyrate, 2-methyl pentanoate, and 3-methyl pentanoate (Fig. 3B \& Table 1). Enzyme activity in the presence of these substrates was similar to hexanoate where activity begins to slow at higher concentrations. Thus, in these instances data were fitted to an equation that incorporated substrate inhibition (See Methods). Utilization of 2ethylbutyrate showed the least product formation $\left(k_{c a t} 6.7 \mathrm{~min}^{-1}\right)$, which may be attributed to the longer side chain (ethyl) compared to 2-methyl butyrate $\left(k_{c a t} 11.3 \mathrm{~min}^{-1}\right)$.

Additionally, while the presence of a methyl branch caused an increase in affinity, it also resulted in decreased product formation as evidenced by lower $k_{c a t}$ values (Fig. 3B \& Table 1). To our knowledge, this is the first demonstration that Buk can utilize unnatural fatty acid precursors capable of altering the membrane fatty acid profile. $L m$ Ptb similarly can utilize unnatural BCCA derivatives as substrates indicating that these two enzymes could likely constitute a pathway to altering membrane composition (Sirobhushanam $e t$ al., 2016). Sen et al. [16] showed that novel fatty acids arising from these unnatural BCCAs imparted the biophysical characteristics necessary for survival of the organism at low temperatures. Our work here indicates that $L$. monocytogenes exploits the reversibility of the Ptb-Buk pathway in the presence of sufficient concentrations of 
unnatural substrates in its environment to supply these fatty acid precursors for elongation by the FAS II pathway. The requirement of higher concentrations in the case of the wild type organism indicates that the domination of Bkd activity in the production of endogenous substrates outcompetes the products from the bypass pathway [11].

\subsection{Buk prefers BCCAs at low temperatures}

L. monocytogenes cld-2/ MOR401 incorporates the products of numerous BCCAs in its membrane at low growth temperatures $[8,16]$. We determined whether Buk was capable of product formation with these substrates at low temperature as would be required if it contributes to increased membrane fluidity at these temperatures. Product formation at $10{ }^{\circ} \mathrm{C}$ was observed with all BCCAs and SCCAs tested (Fig. 3C \& Table 2). Interestingly, substrate inhibition was not observed at low temperatures. We observed lower $k_{c a t}$ values for all of the substrates tested. However, the decrease was more pronounced for SCCAs compared to BCCAs (Fig. 3C \& Table 2), which may be due in part to an increase in the $K_{0.5}$ values for the SCCA substrates. This drastic reduction in Buk catalytic efficiency in the presence of SCCAs compared to BCCAs suggests that Buk demonstrates a strong preference for BCCAs at $10^{\circ} \mathrm{C}$ (Fig. 3C \& Table 2). To our knowledge, this is the first report demonstrating a temperature-dependent switch in substrate preference by Buk from any organism. $\mathrm{LmFabH}$ also demonstrates a switch in its substrate preference at low temperatures to select for 2-methylbutyryl-CoA, the precursor of the low melting point fatty acid anteiso C15:0 enabling it to survive and grow at low temperatures [36]. The substrate specificities of $L m B u k$ could thus be similar to other organisms which increase the membrane BCFAs in response to lower 
temperatures and can incorporate exogenous carboxylic acids. Perhaps these unique substrate specificities are indicative of evolutionary adaptations within L. monocytogenes providing a psychrotolerant selective advantage.

\subsection{Buk catalysis occurs through a ternary complex intermediate}

We sought to determine whether Buk catalysis occurs through a sequential or ping-pong mechanism. Enzyme activity was plotted as a function of butyrate concentration at different fixed concentrations of ATP (i.e. $5 \mathrm{mM}, 7.5 \mathrm{mM}$ and $10 \mathrm{mM}$ ). Double reciprocal plots (Fig. 5) showed that the lines converged to a point on the Y-axis. This is consistent with a reaction mechanism involving a sequential mechanism with the formation of a ternary complex. That is, $L m B$ Buk binds both butyrate and ATP forming a ternary complex, prior to catalysis and release of the products butyryl phosphate and ADP. Additionally, competitive binding of ADP (Fig. 4) in the active site is supportive of the sequential mechanism.

A similar mode of action was also suggested for the action of other phosphokinases such as acetate kinase (Ack) and glycerol kinase [38]. Although the apparent $K_{0.5}$ values and relative activity of Buk with various substrates were explored in C. acetobutylicum and the spirochete MA-2, the mechanism of action was not investigated in these organisms (Hartmanis, 1987; Harwood \& Canale-Parola, 1982). Cheek et al., [39] classified and characterized the protein folding of kinases and showed the similarity of the structure of Ack and Buk. Buk is highly conserved among Listeria isolates and also demonstrates a significant identity with Buk from C. acetobutylicum and Thermotoga maritima. Additionally, substrate and nucleotide bound structures of 
Salmonella typhimurium propionate kinase showed that the carboxyl group of propionate is positioned at a distance of $0.5 \mathrm{~nm}$ from the $\gamma$-phosphate of ATP in the active site supporting a direct in-line transfer mechanism, i.e. a sequential mechanism [40].

\section{Conclusions}

LmBuk demonstrated significant activity with a broad range of SCCAs and BCCA substrates both natural and unnatural. This suggests that Buk might play a role in altering membrane fatty acid composition. Buk is distinct from acetate kinase and is also not involved in the activation of long chain fatty acids. Buk catalysis involves the formation of a ternary complex similar to other members of the phosphokinase protein family. Substantial product formation in the presence of butyrate (present in high concentrations in the mammalian gut) is consistent with a role for Buk in signal transduction during infection in L. monocytogenes.

\section{Acknowledgements}

This work was supported by grant 1 R15 AI099977-01 from the National Institutes of Health to Brian J Wilkinson and Craig Gatto and 1 R15 GM61583 to Craig Gatto. This work was also supported by the R. D. Weigel grant from the Beta Lambda Chapter of the Phi Sigma Biological Honor Society at Illinois State University. The funding sources had no role in study design, collection, analysis and interpretation of data, writing of this manuscript, or the decision to submit it for publication. 


\section{Table 1}

Steady state kinetic analysis of Buk from L. monocytogenes at $37^{\circ} \mathrm{C}$

\begin{tabular}{llll}
\hline Substrate & $k_{\text {cat }}\left(\mathrm{min}^{-1}\right)$ & $K_{M}(\mathrm{mM})$ & $\begin{array}{l}k_{\text {cat }} / K_{M}\left(\mathrm{mM}^{-}\right. \\
\left.\mathrm{min}^{-1}\right)\end{array}$ \\
\hline Straight-chain & & \\
& & & \\
Propionate & $155.6 \pm 0$ & $174.1 \pm 68.9$ & 0.89 \\
Butyrate & $50.2 \pm 0.4$ & $50.5 \pm 5.8$ & 0.99 \\
Pentanoate & $71.7 \pm 2.2$ & $13.1 \pm 3.3$ & 5.5 \\
Hexanoate & $1310.9 \pm 474.9$ & $1856 \pm 676$ & 0.7 \\
& & & \\
Branched-chain & & & \\
& & & \\
Iso butyrate & $23.7 \pm 0.4$ & $7.6 \pm 1.3$ & 3.1 \\
Isovalerate & $25 \pm 0.1$ & $57.1 \pm 9.6$ & 0.44 \\
2-methylbutyrate & $15.9 \pm 1.5$ & $36.2 \pm 5.2$ & 0.44 \\
2-ethyl butyrate & $6.7 \pm 1.7$ & $16.5 \pm 0.9$ & 0.4 \\
2-methyl pentanoate & $18.8 \pm 2.4$ & $44.2 \pm 8.5$ & 0.4 \\
3-methyl pentanoate & $21.2 \pm 0.4$ & $27.3 \pm 0.8$ & 0.8 \\
\hline
\end{tabular}

Initial velocities were measured at $37^{\circ} \mathrm{C}$ in the presence of $10 \mathrm{mM}$ ATP at $\mathrm{pH}$ 7.5. The $k_{c a t}$ and $K_{M}$ were calculated from the least squares fit of the experimental data from the different substrates to the Michaelis-Menten equation. The values indicated are the mean of experiments performed at least in triplicate \pm SEM. 


\section{Table 2}

Steady state kinetic analysis of Buk from L. monocytogenes at $10^{\circ} \mathrm{C}$

\begin{tabular}{llll}
\hline Substrate & $k_{\text {cat }}\left(\mathrm{min}^{-1}\right)$ & $K_{M}(\mathrm{mM})$ & $\begin{array}{l}k_{\text {cat }} / K_{M} \\
\left(\mathrm{mM}^{-1} \mathrm{~min}^{-1}\right)\end{array}$ \\
\hline Straight-chain & & & \\
Butyrate & $8.2 \pm 0.1$ & $78.1 \pm 3.8$ & 0.1 \\
Pentanoate & $9.1 \pm 0.4$ & $42.5 \pm 5.2$ & 0.2 \\
& & & \\
Branched-chain & & & \\
& & & \\
Isobutyrate & $10.2 \pm 0.3$ & $9 \pm 1.5$ & 1.1 \\
Isovalerate & $7.6 \pm 0.3$ & $8.2 \pm 1.4$ & 0.9 \\
2-methylbutyrate & $4.3 \pm 0.2$ & $22 \pm 4.3$ & 0.2 \\
2-ethyl butyrate & $1.2 \pm 0.1$ & $4 \pm 2.5$ & 0.3 \\
\hline
\end{tabular}

Initial velocities were measured at $10^{\circ} \mathrm{C}$ in the presence of $10 \mathrm{mM}$ ATP at $\mathrm{pH}$ 7.5. The $k_{c a t}$ and $K_{M}$ were calculated from the least squares fit of the experimental data from the different substrates to the Michaelis-Menten equation. The values indicated are the mean of experiments performed at least in triplicate \pm SEM. 


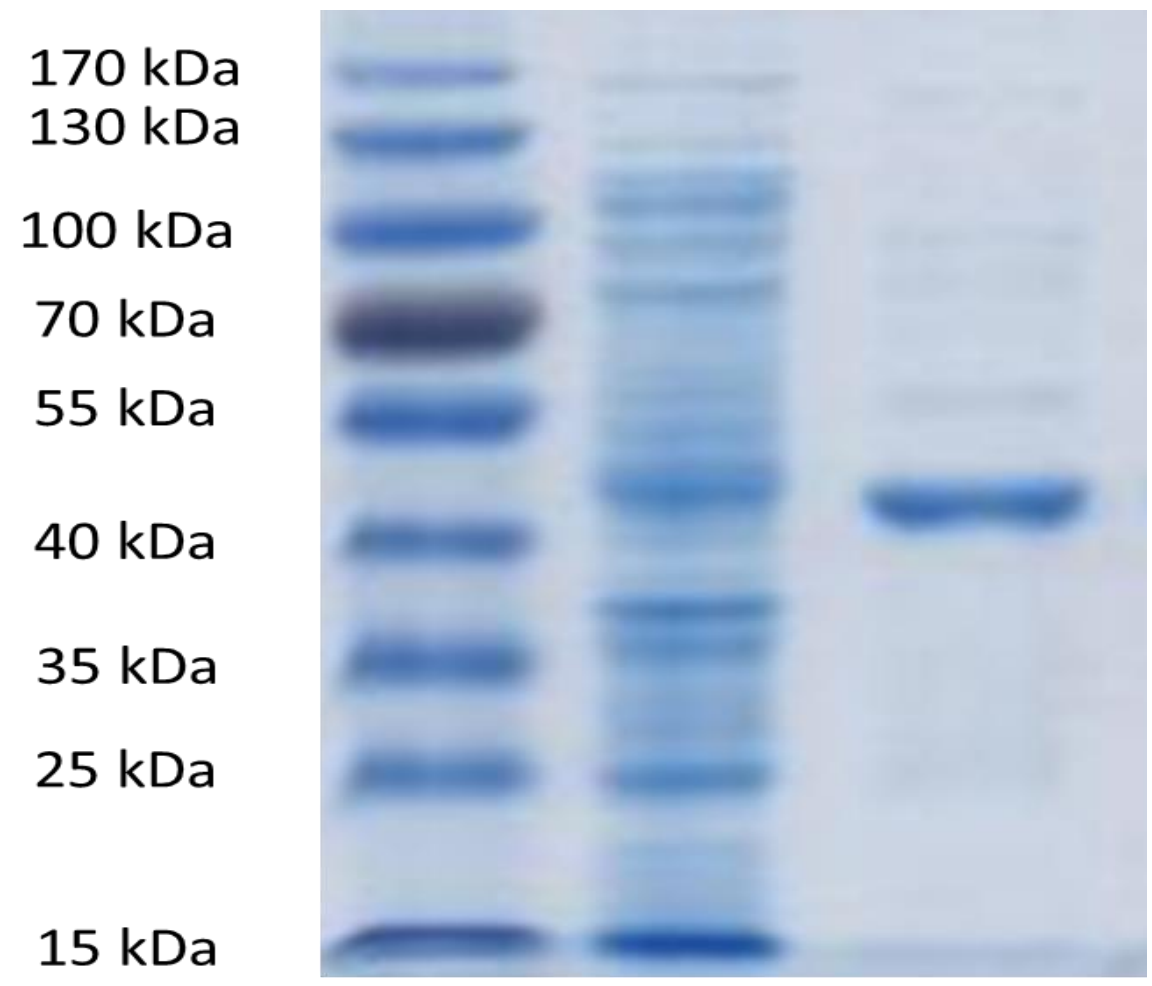

Fig. 1. Analysis of purified $L m B$ Buk by SDS-PAGE. Samples were resolved on $10 \%$ (w/v) acrylamide gel and stained with Coomassie Blue. Lane 1 protein molecular weight markers, Lane 2 Cell-free lysate showing expression of LmBuk, Lane 3 Recombinant $L m$ Buk after purification by $\mathrm{Ni}^{2+}$ - affinity chromatography. 


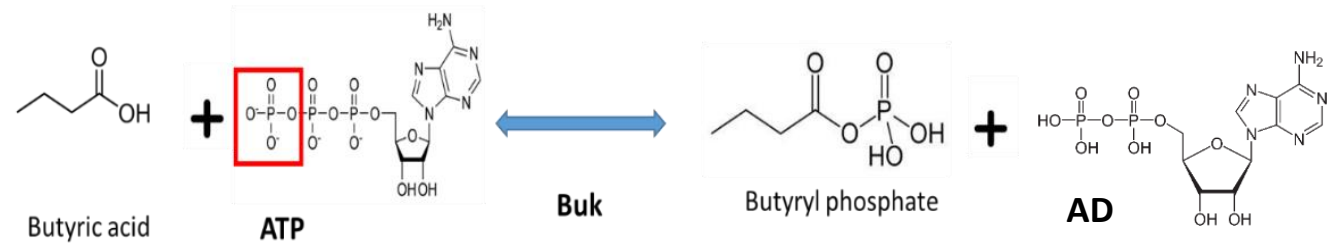

Step 2

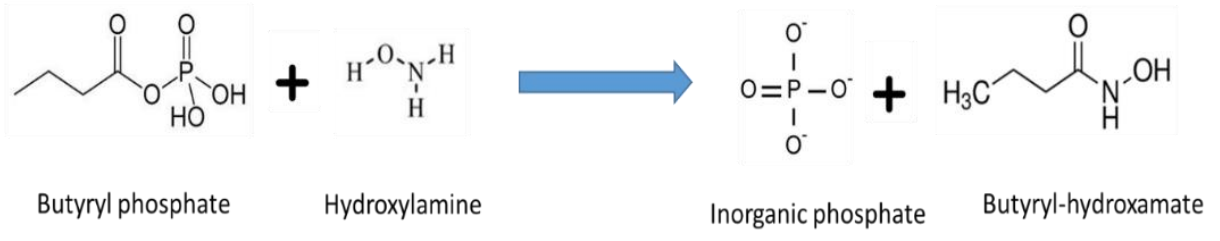

Fig. 2. Reaction catalyzed by $L m B u k$. Step 1 shows the activity of $L m B$ uk catalyzing the reversible transfer of the terminal phosphate moiety of ATP (highlighted in red) to the carbonyl group of butyric acid resulting in the formation of butyryl phosphate. Step 2 shows the irreversible conversion of the unstable butyryl phosphate to stable butyryl hydroxamate with the release of inorganic phosphate. Butyryl hydroxamate in the presence of acidic ferric chloride forms a purple complex which is quantified at $540 \mathrm{~nm}$. 


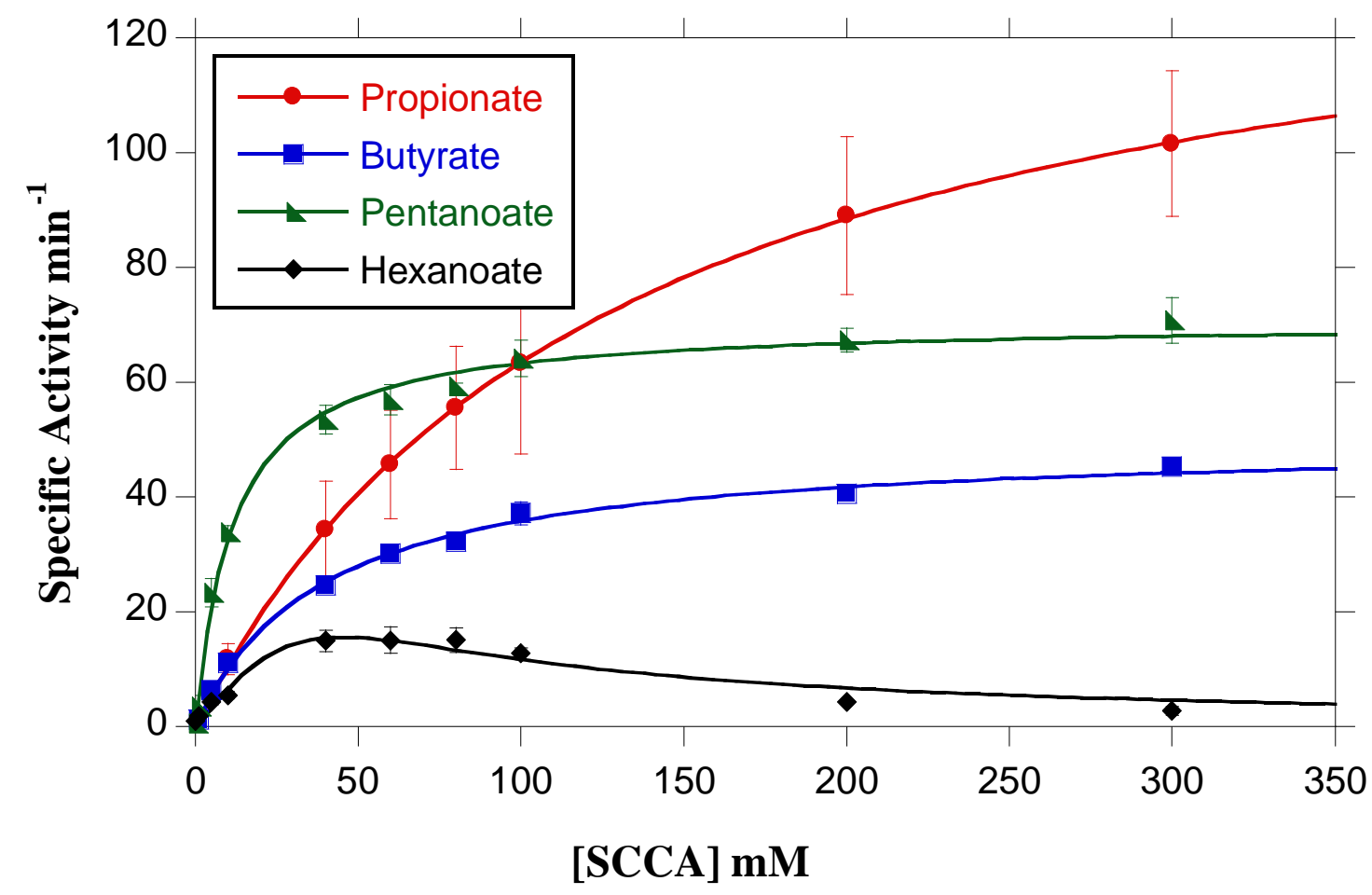

Fig. 3. Substrate preference of $L m B u k$. A. Concentration-dependent activity of $L m B u k$ in the presence of SCCA substrates at $37^{\circ} \mathrm{C}$. The observed initial rates of reaction for the substrates propionate (closed circles), butyrate (closed squares), pentanoate (closed triangles) and hexanoate (closed diamonds) catalyzed by $\operatorname{LmBuk}$ were determined at 37 ${ }^{\circ} \mathrm{C}$ in the presence of $10 \mathrm{mM}$ ATP under standard assay conditions (see Materials and Methods). Data were plotted as a function of the substrate concentration and $K_{M}$ and $k_{c a t}$ were determined from the fit of the data to the Michaelis-Menten equation using Kaleidagraph software. Data derived for hexanoate was fitted to the Michaelis-Menten equation to include substrate inhibition. Data are means \pm SEM of at least three experiments. 


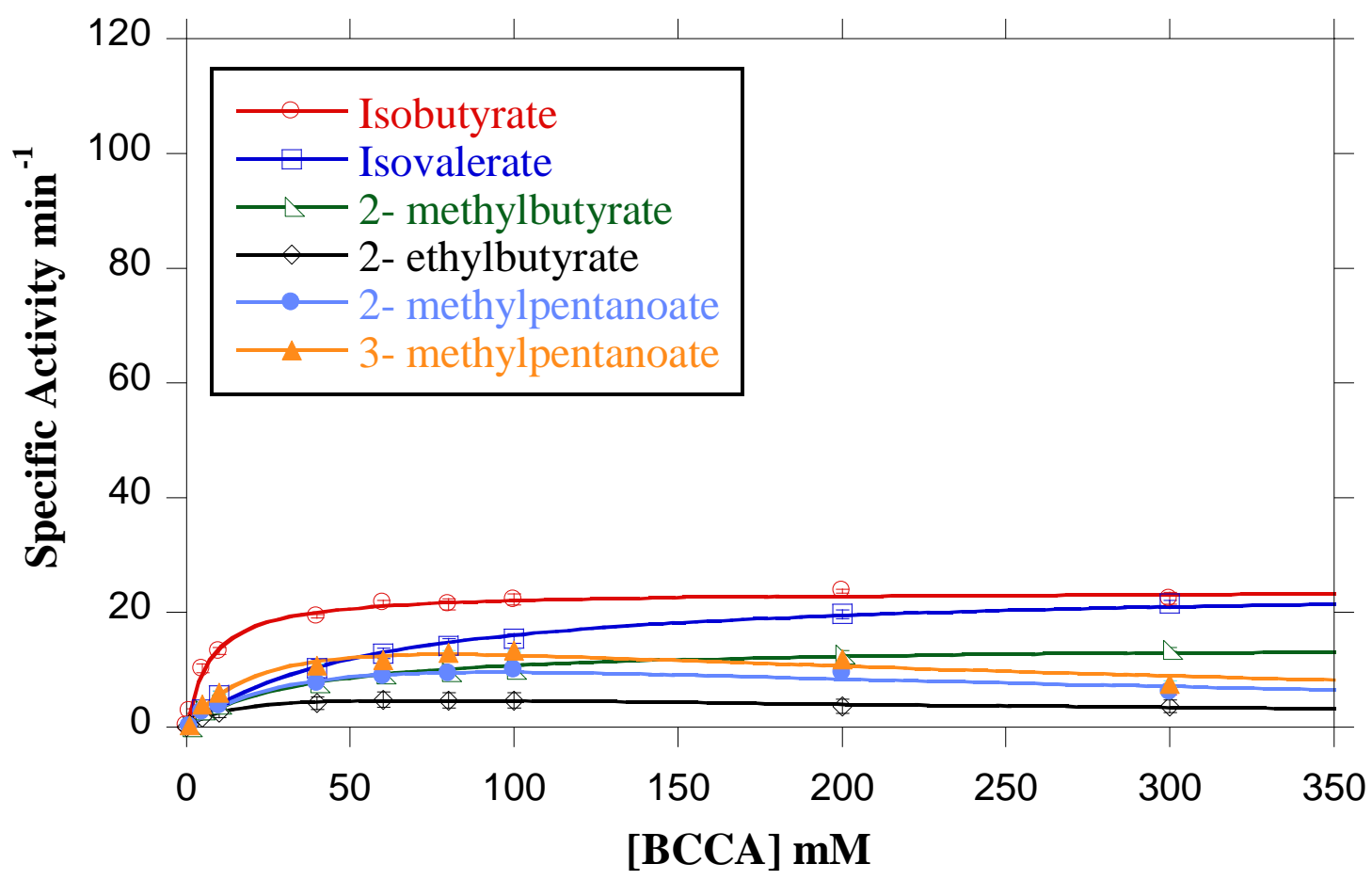

Fig. 3B. Concentration-dependent activity of $L m B u k$ in the presence of BCCA substrates at $37{ }^{\circ} \mathrm{C}$. The observed initial rates of reaction for the isobutyrate (open circles), isovalerate (open squares), 2-methyl butyrate (open triangles) and 2-ethyl butyrate (open diamonds), 2-methyl pentanoate (closed circles) and 3-methyl pentanoate (closed triangles) catalyzed by $\mathrm{LmBuk}$ were determined at $37^{\circ} \mathrm{C}$ in the presence of $10 \mathrm{mM}$ ATP under standard assay conditions (see Materials and Methods). Data were plotted as a function of the substrate concentration and $K_{M}$ and $k_{c a t}$ were determined from the fit of the data to the Michaelis-Menten equation using Kaleidagraph software. Data derived for 2-ethylbutyrate, 2-methylpentanoate and 3-methylpentanoate was fitted to the MichaelisMenten equation to include substrate inhibition. Data are means \pm SEM of at least three experiments. 


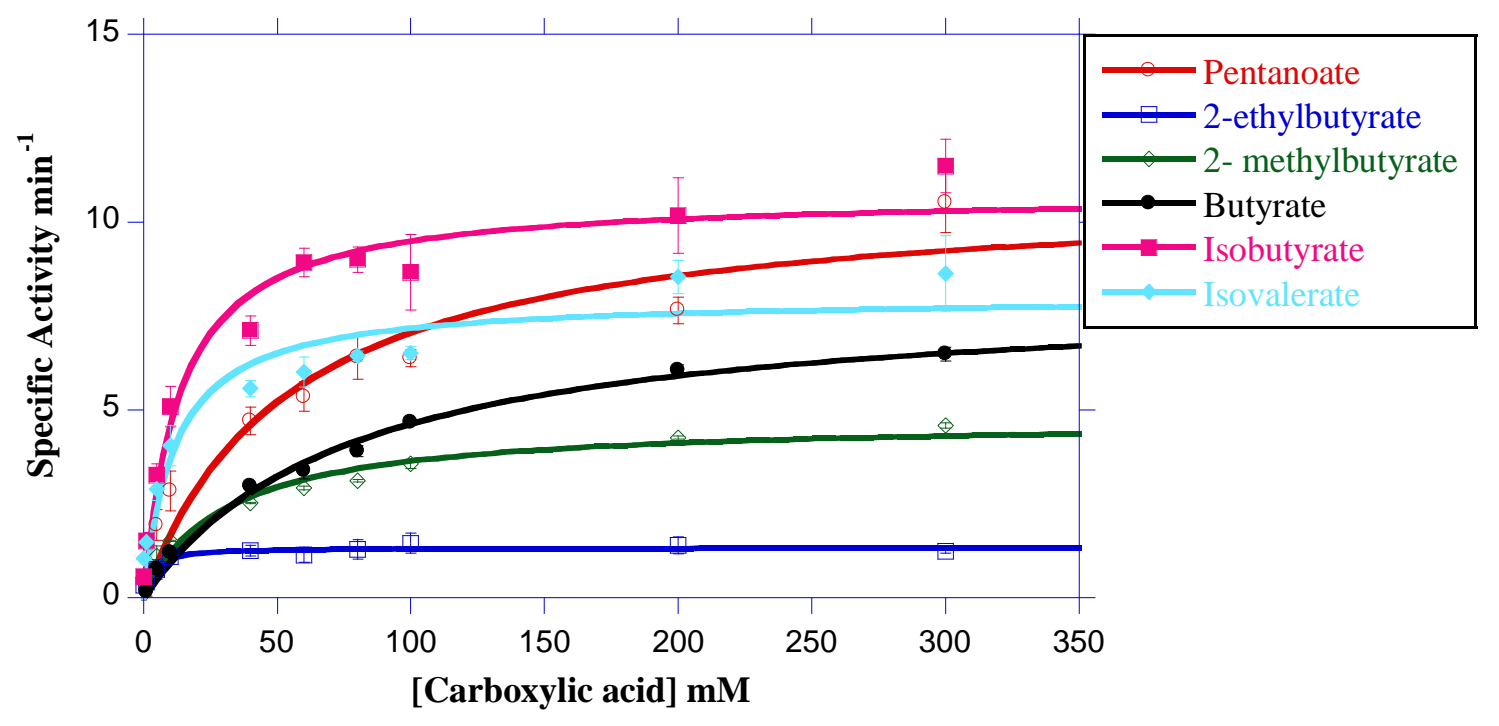

Fig. 3C. Concentration-dependent activity of $\operatorname{LmBuk}$ at $10{ }^{\circ} \mathrm{C}$ in the presence of SCCA and BCCA substrates. The observed initial rates of reaction for the isobutyrate (closed squares), isovalerate (closed diamonds), 2-methyl butyrate (open diamonds) and 2-ethyl butyrate (open squares), pentanoate (open circles) and butyrate (closed circles) catalyzed by $L m$ Buk were determined at $10{ }^{\circ} \mathrm{C}$ in the presence of $10 \mathrm{mM}$ ATP as described under "Materials and Methods". Data were plotted as a function of the substrate concentration and $K_{M}$ and $k_{\text {cat }}$ were determined from the fit of the data to the Michaelis-Menten equation using Kaleidagraph software. Data plotted are means \pm SEM of at least three experiments. 


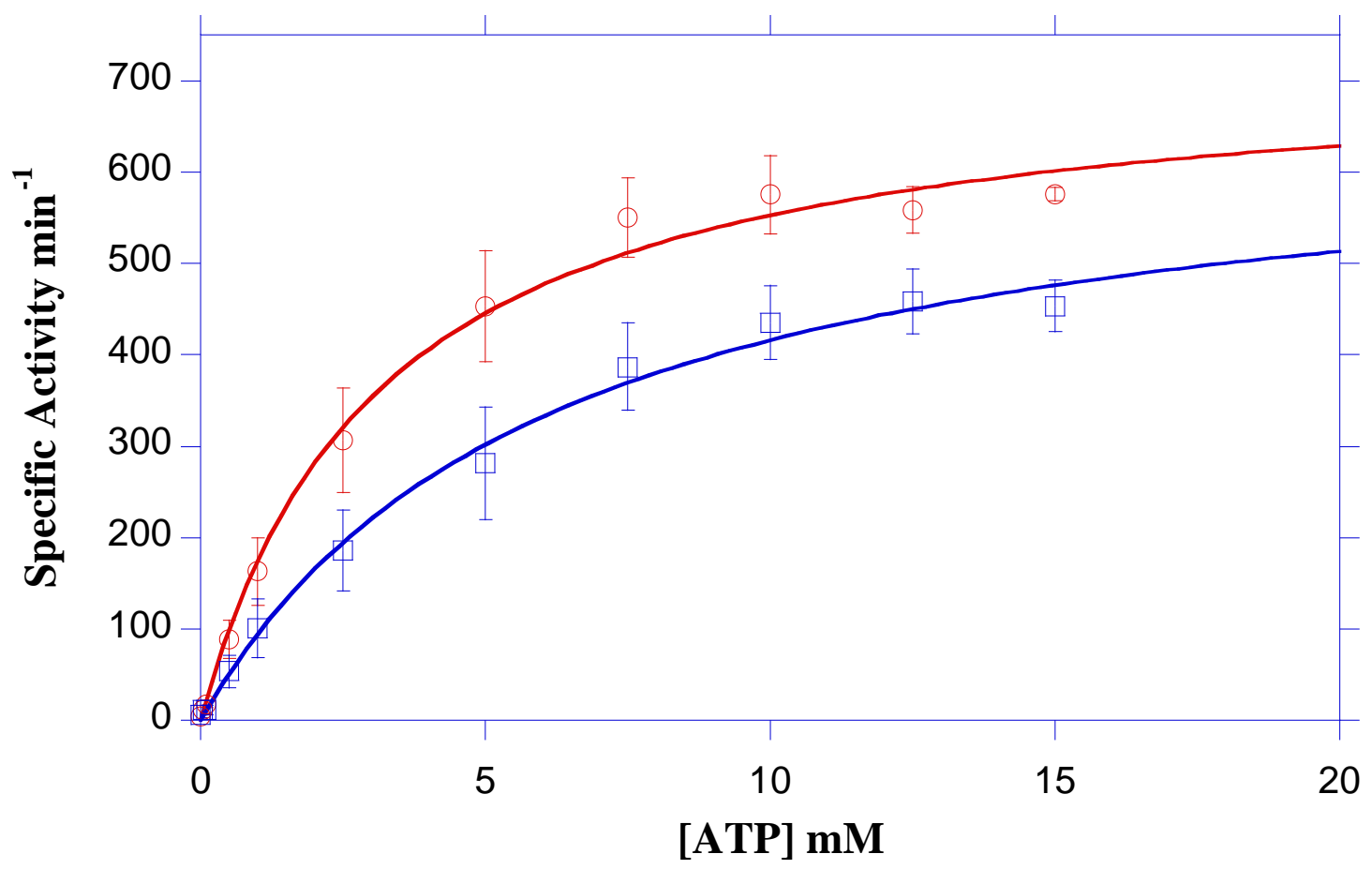

Fig. 4. Concentration dependence of ATP utilization by $L m B u k$ and competitive inhibition by ADP. $L m$ Buk activity was determined under standard assay conditions in the presence of $200 \mathrm{mM}$ butyrate and varying amounts of ATP $(10 \mu \mathrm{M}-15 \mathrm{mM})$ and data (open circles) were plotted as a function of ATP concentration. Fit of the data to the Michaelis-Menten equation provided values for $K_{M}$. ADP inhibition studies were performed using $2 \mathrm{mM}$ ADP in each reaction under the same experimental conditions as that of the ATP assay (open squares). ADP demonstrated competitive inhibition with respect to ATP. Data plotted are means \pm SEM of at least three experiments. 


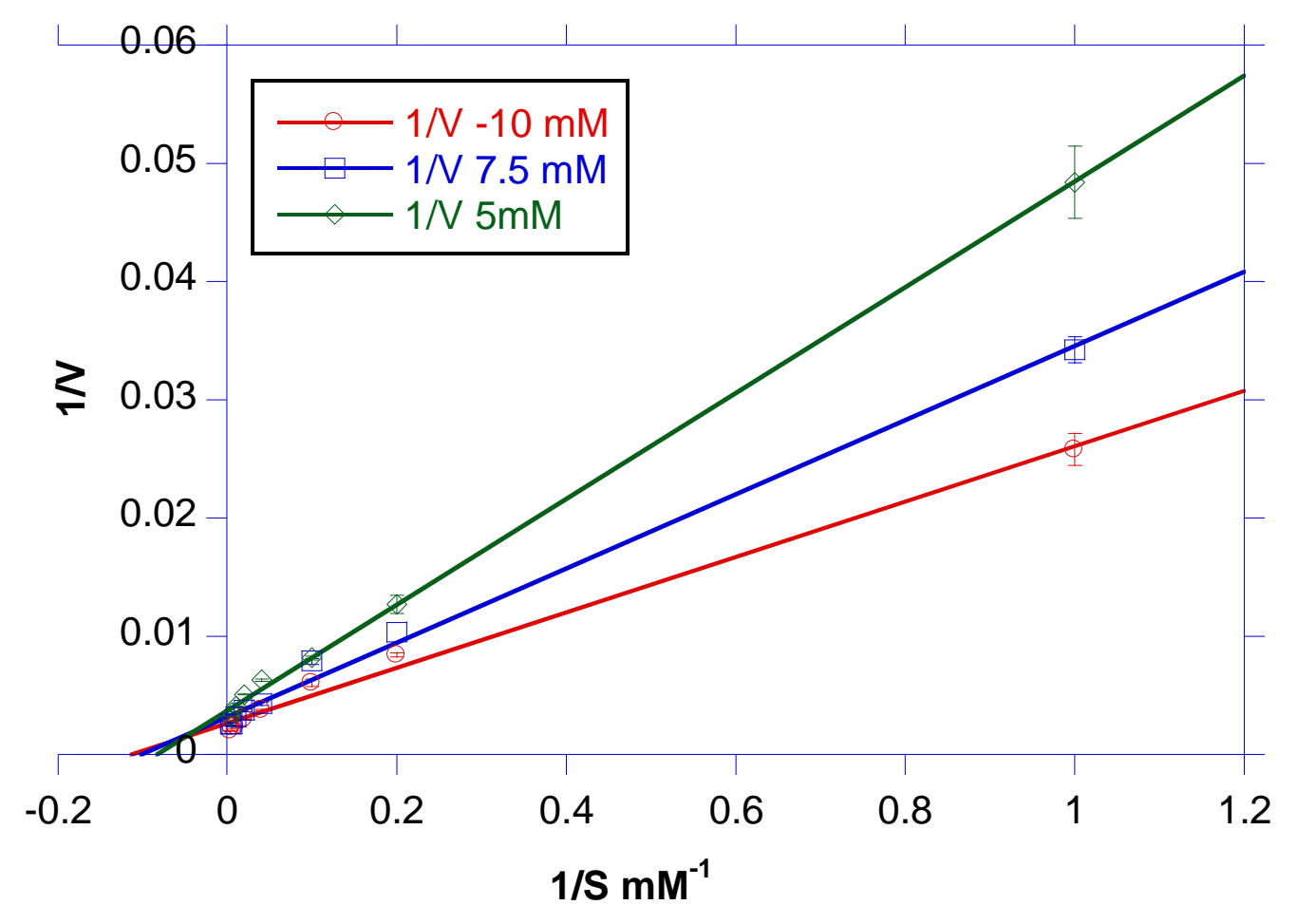

Fig. 5. $L m B$ Buk catalysis occurs through a ternary complex intermediate. Concentration dependent activity of $L m$ Buk in the presence of varying ATP concentrations, $5 \mathrm{mM}$ (open diamonds), $7.5 \mathrm{mM}$ (open squares) and $10 \mathrm{mM}$ (open circles) and varying concentrations of butyrate $(0.1-300 \mathrm{mM})$ were determined under standard assay conditions at $37{ }^{\circ} \mathrm{C}$. Means of specific activity from at least two experiments \pm SEM were plotted. 


\section{References}

[1] P. Cossart, Illuminating the landscape of host-pathogen interactions with the bacterium Listeria monocytogenes, Proc. Natl. Acad. Sci., 108 (2011) 19484-19491.

[2] S.J. Walker, P. Archer, J.G. Banks, Growth of Listeria monocytogenes at refrigeration temperatures, J. Appl. Bacteriol., 68 (1990) 157-162.

[3] D. Poger, B. Caron, A.E. Mark, Effect of methyl-branched fatty acids on the structure of lipid bilayers, J. Phys. Chem., 118 (2014) 13838-13848.

[4] B.A. Annous, L.A. Becker, D.O. Bayles, D.P. Labeda, B.J. Wilkinson, Critical role of anteiso-C15:0 fatty acid in the growth of Listeria monocytogenes at low temperatures, Appl. Environ. Microbiol., 63 (1997) 3887-3894.

[5] M.R. Edgcomb, S. Sirimanne, B.J. Wilkinson, P. Drouin, R.D. Morse, Electron paramagnetic resonance studies of the membrane fluidity of the foodborne pathogenic psychrotroph Listeria monocytogenes, Biochim. Biophys. Acta, 1463 (2000) 31-42.

[6] M. Sinensky, Homeoviscous adaptation--a homeostatic process that regulates the viscosity of membrane lipids in Escherichia coli, Proc. Natl. Acad. Sci. U. S. A., 71 (1974) 522-525.

[7] H. Oku, T. Kaneda, Biosynthesis of branched-chain fatty acids in Bacillus subtilis. A decarboxylase is essential for branched-chain fatty acid synthetase, J. Biol. Chem., 263 (1988) 18386-96.

[8] K. Zhu, D.O. Bayles, A. Xiong, R.K. Jayaswal, B.J. Wilkinson, Precursor and temperature modulation of fatty acid composition and growth of Listeria monocytogenes cold-sensitive mutants with transposon-interrupted branched-chain alpha-keto acid dehydrogenase, Microbiology, 151 (2005) 615-23.

[9] Y. Sun, M.X.D. O'Riordan, Branched-chain fatty acids promote Listeria monocytogenes intracellular infection and virulence, Infect. Immun., 78 (2010) 4667-4673.

[10] Y. Sun, B.J. Wilkinson, T.J. Standiford, H.T. Akinbi, M.X.D. O’Riordan, Fatty acids regulate stress resistance and virulence factor production for Listeria monocytogenes, J. Bacteriol., 194 (2012) 5274-5284.

[11] M. Julotok, A.K. Singh, C. Gatto, B.J. Wilkinson, Influence of fatty acid precursors, including food preservatives, on the growth and fatty acid composition of Listeria monocytogenes at 37 and 10degreesC, Appl. Environ. Microbiol., 76 (2010) 142332.

[12] E.S. Giotis, D. a. McDowell, I.S. Blair, B.J. Wilkinson, Role of branched-chain fatty acids in $\mathrm{pH}$ stress tolerance in Listeria monocytogenes, Appl. Environ. Microbiol., 73 (2007) 997-1001.

[13] V.K. Singh, D.S. Hattangady, E.S. Giotis, A.K. Singh, N.R. Chamberlain, M.K. Stuart, B.J. Wilkinson, Insertional inactivation of branched-chain alpha-keto acid dehydrogenase in Staphylococcus aureus leads to decreased branched-chain membrane fatty acid content and increased susceptibility to certain stresses, Appl. Environ. Microbiol., 74 (2008) 5882-90. 
[14] K. Willecke, A.B. Pardee, Fatty acid-requiring mutant of Bacillus subtilis defective in branched chain alpha-keto acid dehydrogenase, J. Biol. Chem., 246 (1971) 526472.

[15] T. Kaneda, Incorporation of branched-chain C6 -fatty acid isomers into the related long-chain fatty acids by growing cells of Bacillus subtilis, Biochemistry, 10 (1970) 340-347.

[16] S. Sen, S. Sirobhushanam, M.P. Hantak, P. Lawrence, J. Thomas Brenna, C. Gatto, B.J. Wilkinson, Short branched-chain C6 carboxylic acids result in increased growth, novel "unnatural" fatty acids and increased membrane fluidity in a Listeria monocytogenes branched-chain fatty acid-deficient mutant, Biochim. Biophys. Acta - Mol. Cell Biol. Lipids, 1851 (2015) 1406-1415.

[17] T. Kaneda, Iso- and anteiso-fatty acids in bacteria: biosynthesis, function, and taxonomic significance, Microbiol. Rev., 55 (1991) 288-302.

[18] D.E. Ward, C.C. van Der Weijden, M.J. van Der Merwe, H. V Westerhoff, A. Claiborne, J.L. Snoep, Branched-chain alpha-keto acid catabolism via the gene products of the bkd operon in Enterococcus faecalis: a new, secreted metabolite serving as a temporary redox sink, J. Bacteriol., 182 (2000) 3239-3246.

[19] K. Zhu, X. Ding, M. Julotok, B.J. Wilkinson, Exogenous isoleucine and fatty acid shortening ensure the high content of anteiso-C15:0 fatty acid required for lowtemperature growth of Listeria monocytogenes, Appl. Environ. Microbiol., 71 (2005) 8002-8007.

[20] K.E. Nelson, D.E. Fouts, E.F. Mongodin, J. Ravel, R.T. DeBoy, J.F. Kolonay, D.A. Rasko, S. V Angiuoli, S.R. Gill, I.T. Paulsen, J. Peterson, O. White, W.C. Nelson, W. Nierman, M.J. Beanan, L.M. Brinkac, S.C. Daugherty, R.J. Dodson, A.S. Durkin, R. Madupu, et al., Whole genome comparisons of serotype $4 \mathrm{~b}$ and 1/2a strains of the food-borne pathogen Listeria monocytogenes reveal new insights into the core genome components of this species, Nucleic Acids Res., 32 (2004) 2386-2395.

[21] D.P. Wiesenborn, F.B. Rudolph, E.T. Papoutsakis, Phosphotransbutyrylase from Clostridium acetobutylicum ATCC 824 and its role in acidogenesis, Appl. Environ. Microbiol., 55 (1989) 317-322.

[22] C.S. Harwood, E. Canale-Parola, Properties of acetate kinase isozymes and a branched-chain fatty acid kinase from a Spirochete, J. Bacteriol., 152 (1982) 246254.

[23] M.G.N. Hartmanis, Butyrate kinase from Clostridium acetobutylicum, J. Biol. Chem., 262 (1987) 617-621.

[24] D.E. Ward, R.P. Ross, C.C. van der Weijden, J.L. Snoep, A. Claiborne, Catabolism of branched-chain alpha-keto acids in Enterococcus faecalis: the bkd gene cluster, enzymes, and metabolic route, J. Bacteriol., 181 (1999) 5433-5442.

[25] F. Lipmann, L.C. Tuttle, A specific micromethod for the determination of acyl phosphates, J. Biol. Chem., 159 (1945) 21-28.

[26] T.L. Romick, H.P. Fleming, R.F. McFeeters, Aerobic and anaerobic metabolism of Listeria monocytogenes in defined glucose medium, Appl. Environ. Microbiol., 62 (1996) 304-7. 
[27] O. Tokarskyy, D.L. Marshall, Mechanism of synergistic inhibition of Listeria monocytogenes growth by lactic acid, monolaurin, and nisin, Appl. Environ. Microbiol., 74 (2008) 7126-9.

[28] S. Sirobhushanam, C. Galva, S. Sen, B.J. Wilkinson, C. Gatto, Broad substrate specificity of phosphotransbutyrylase from Listeria monocytogenes: A potential participant in an alternative pathway for provision of acyl CoA precursors for fatty acid biosynthesis, BBA - Mol. Cell Biol. Lipids, 1861 (2016) 1102-1110.

[29] S.J. Liu, A Steinbüchel, Exploitation of butyrate kinase and phosphotransbutyrylase from Clostridium acetobutylicum for the in vitro biosynthesis of poly(hydroxyalkanoic acid), Appl. Microbiol. Biotechnol., 53 (2000) 545-52.

[30] G.J. Vazquez, M.J. Pettinari, B.S. Méndez, Evidence of an association between poly(3-hydroxybutyrate) accumulation and phosphotransbutyrylase expression in Bacillus megaterium, Int. Microbiol., 6 (2003) 127-129.

[31] T. Tobe, N. Nakanishi, N. Sugimoto, Activation of motility by sensing short-chain fatty acids via two steps in a flagellar gene regulatory cascade in enterohemorrhagic Escherichia coli, Infect. Immun., 79 (2011) 1016-1024.

[32] I. Gantois, R. Ducatelle, F. Pasmans, F. Haesebrouck, A. Thompson, J.C. Hinton, F. Van Immerseel, I. Hautefort, Butyrate specifically down-regulates Salmonella Pathogenicity Island 1 Gene Expression, Appl. Environ. Microbiol., 72 (2006) 946949.

[33] Y. Zhao, C.A. Tomas, F.B. Rudolph, E.T. Papoutsakis, G.N. Bennett, Intracellular butyryl phosphate and acetyl phosphate concentrations in Clostridium acetobutylicum and their implications for solvent formation, Appl. Environ. Microbiol., 71 (2005) 530-537.

[34] W.R. McCleary, J.B. Stock, Acetyl phosphate and the activation of two-component response regulators, J. Biol. Chem., 269 (1994) 31567-31572.

[35] J.H. Cummings, E.W. Pomare, W.J. Branch, C.P. Naylor, G.T. Macfarlane, Short chain fatty acids in human large intestine, portal, hepatic and venous blood, Gut, 28 (1987) 1221-1227.

[36] A.K. Singh, Y. Zhang, K. Zhu, C. Subramanian, Z. Li, K. Jayaswal, C. Gatto, C.O. Rock, B.J. Wilkinson, FabH selectivity for anteiso branched-chain fatty acid precursors in low temperature adaptation in Listeria monocytogenes, FEMS Microbiol. Lett., 301 (2009) 1-8.

[37] K.-H.H.K. Choi, R.J. Heath, C.O. Rock, $\beta$-Ketoacyl-acyl carrier protein synthase III $(\mathrm{FabH})$ is a determining factor in branched-chain fatty acid biosynthesis, $\mathrm{J}$. Bacteriol., 182 (2000) 365-370.

[38] W.A. Blattler, J.R. Knowles, Stereochemical course of phosphokinases. The use of adenosine $[\mathrm{g}-(\mathrm{S})-16 \mathrm{O}, 17 \mathrm{O}, 18 \mathrm{O}]$ triphosphate and the mechanistic consequences for the reactions catalyzed by glycerol kinase, hexokinase, pyruvate kinase, and acetate kinase, Biochemistry, 18 (1979) 3927-3933.

[39] S. Cheek, H. Zhang, N. V. Grishin, Sequence and structure classification of kinases, J. Mol. Biol., 320 (2002) 855-881.

[40] A.M.V. Murthy, S. Mathivanan, S. Chittori, H.S. Savithri, M.R.N. Murthy, Structures of substrate- and nucleotide-bound propionate kinase from Salmonella 
typhimurium: substrate specificity and phosphate-transfer mechanism, Acta Crystallogr. Sect. D Biol. Crystallogr., 71 (2015) 1640-1648. 


\section{CHAPTER III}

\section{SUMMARY}

The high membrane BCFA content is essential for the fitness and virulence of $L$. monocytogenes and is influenced by the strong affinity of the FabH enzyme towards branched-chain acyl CoA substrates. In addition, presence of unnatural BCCA substrates which fulfill the structural requirements of branch position result in incorporation of novel BCFAs in the membrane. Although the presence of an alternate pathway converting the BCCAs into their acyl CoA derivatives capable of entering the fatty acid biosynthesis pathway is the logical conclusion from these observations, the enzymes involved in this pathway have not been identified in L. monocytogenes. Ptb and Buk, whose activities resemble that of phosphotransacetylase (pta) and acetate kinase (ack) respectively, were concluded to be the most likely candidates due to their reported ability to utilize multiple substrates in other organisms, reversibility of their catalytic activity, and their location in the $b k d$ operon. Ptb from L. monocytogenes demonstrated broad substrate specificity and especially prefers substrates with a carbon chain length of C3-C5 and the presence of an alkyl side chain. Ptb also exhibited activity with natural SCCAs and BCCAs and also with unnatural substrates such as 2-ethyl butyrate, that are capable of altering the membrane fatty acid composition. On the other hand, Buk from $L$. monocytogenes demonstrated significant activity with a broad range of SCCAs and 
BCCA substrates both natural and unnatural such as 2-ethyl butyrate. Buk is distinct from acetate kinase and is also not involved in the activation of long chain fatty acids. Buk catalysis involves the formation of a ternary complex similar to other phosphokinases. This suggests that Ptb and Buk together likely play an important role in the activation of natural and unnatural carboxylic acids with their subsequent incorporation into the membrane fatty acids after elongation. The high activity of Ptb with butyryl CoA, and Buk with butyrate indicates that it could be involved in signal processing upon exposure of L. monocytogenes to butyrate. Butyrate also probably plays a significant role in the regulation of gene expression, since exposure to butyrate leads to decreased expression of virulence genes in L. monocytogenes. Additionally, high concentrations of butyrate also lead to diminished growth of L. monocytogenes due to the incorporation of SCFAs in the membrane, suggesting a possible control strategy for the organism. Although we were able to demonstrate the strong likelihood of the involvement of this pathway in the activation of carboxylic acids, creation of a knockout mutant of this pathway in $L$. monocytogenes and further analysis of supplementation of BCCAs in the mutant could yield conclusive evidence of its role. Furthermore, the limited presence of this pathway makes it essential to understand its role in vivo and during virulence due to the high concentration of short chain fatty acids in the human gut. 